\title{
Turán problems on Non-uniform Hypergraphs
}

\author{
J. Travis Johnston \\ Department of Mathematics \\ University of South Carolina \\ Columbia, South Carolina, U.S.A. \\ j.travis.johnston@gmail.com
}

\author{
Linyuan $\mathrm{Lu}^{*}$ \\ Department of Mathematics \\ University of South Carolina \\ Columbia, South Carolina, U.S.A. \\ lu@math.sc.edu
}

Submitted: Nov 25, 2013; Accepted: Oct 22, 2014; Published: Oct 30, 2014

Mathematics Subject Classifications: 05D05, 05C65, 05D40

\begin{abstract}
A non-uniform hypergraph $H=(V, E)$ consists of a vertex set $V$ and an edge set $E \subseteq 2^{V}$; the edges in $E$ are not required to all have the same cardinality. The set of all cardinalities of edges in $H$ is denoted by $R(H)$, the set of edge types. For a fixed hypergraph $H$, the Turán density $\pi(H)$ is defined to be $\lim _{n \rightarrow \infty} \max _{G_{n}} h_{n}\left(G_{n}\right)$, where the maximum is taken over all $H$-free hypergraphs $G_{n}$ on $n$ vertices satisfying $R\left(G_{n}\right) \subseteq R(H)$, and $h_{n}\left(G_{n}\right)$, the so called Lubell function, is the expected number of edges in $G_{n}$ hit by a random full chain. This concept, which generalizes the Turán density of $k$-uniform hypergraphs, is motivated by recent work on extremal poset problems. The details connecting these two areas will be revealed in the end of this paper.

Several properties of Turán density, such as supersaturation, blow-up, and suspension, are generalized from uniform hypergraphs to non-uniform hypergraphs. Other questions such as "Which hypergraphs are degenerate?" are more complicated and don't appear to generalize well. In addition, we completely determine the Turán densities of $\{1,2\}$-hypergraphs.
\end{abstract}

Keywords: non-uniform hypegraphs, Turán density, poset, diamond conjecture, R-graphs

\section{Introduction}

A hypergraph $H$ is a pair $(V, E)$; $V$ is the vertex set, and $E \subseteq 2^{V}$ is the edge set. If all edges have the same cardinality $k$, then $H$ is a $k$-uniform hypergraph. Turán problems on $k$-uniform hypergraphs have been actively studied for many decades. However,

\footnotetext{
*This author was supported in part by NSF grant DMS 1300547 and ONR grant N00014-13-1-0717.
} 
Turán problems on non-uniform hypergraphs are rarely considered (see [40, 35] for two different treatments). Very recently, several groups of people have started actively studying extremal families of sets avoiding given sub-posets. Several new problems have been established. One of them is the diamond problem:

The diamond conjecture: [27] Any family $\mathcal{F}$ of subsets of $[n]:=\{1,2, \ldots, n\}$ with no four sets $A, B, C, D$ satisfying $A \subseteq B \cap C, B \cup C \subseteq D$ can have at most $(2+o(1))\left(\begin{array}{c}n \\ \left\lfloor\frac{n}{2}\right\rfloor\end{array}\right)$ subsets.

This conjecture, along with many other problems, motivates us to study Turán-type problems on non-uniform hypergraphs. The details of this connection will be given in the last section.

We briefly review the history of Turán Problems on uniform hypergraphs. Given a positive integer $n$ and a $k$-uniform hypergraph $H$ on $n$ vertices (or $k$-graph, for short), the Turán number ex $(n, H)$ is the maximum number of edges in a $k$-graph on $n$ vertices that does not contain $H$ as a subgraph; such a graph is called $H$-free. Katona et al. 28] showed that $f(n, H)=\operatorname{ex}(n, H) /\left(\begin{array}{l}n \\ k\end{array}\right)$ is a decreasing function of $n$. The limit $\pi(H)=\lim _{n \rightarrow \infty} f(n, H)$, which always exists, is called the Turán density of $H$.

For $k=2$, the graph case, Erdös-Stone-Simonovits proved $\pi(G)=1-\frac{1}{\chi(G)-1}$ for any graph $G$ with chromatic number $\chi(G) \geqslant 3$. If $G$ is bipartite, then $\operatorname{ex}(n, G)=o\left(n^{2}\right)$. The magnitude of $\operatorname{ex}(n, G)$ is unknown for most bipartite graphs $G$.

Let $K_{k}^{r}$ denote the complete $r$-graph on $k$ vertices. Turán determined the value of $\operatorname{ex}\left(n, K_{k}^{2}\right)$ which implies that $\pi\left(K_{k}^{2}\right)=1-\frac{1}{k-1}$ for all $k \geqslant 3$. However, no Turán density $\pi\left(K_{k}^{r}\right)$ is known for any $k>r \geqslant 3$. The most extensively studied case is when $k=4$ and $r=3$. Turán conjectured [50] that $\pi\left(K_{4}^{3}\right)=5 / 9$. Erdös [15] offered $\$ 500$ for determining any $\pi\left(K_{k}^{r}\right)$ with $k>r \geqslant 3$ and $\$ 1000$ for answering it for all $k$ and $r$. The upper bounds for $\pi\left(K_{4}^{3}\right)$ have been sequentially improved: 0.6213 (de Caen [11]), 0.5936 (Chung-Lu [6]), 0.56167 (Razborov [42], using the flag algebra method). There are a few uniform hypergraphs whose Turán density has been determined: the Fano plane [20, 32], expanded triangles [33], 3-books, 4-books [19], $F_{5}$ [17], extended complete graphs [41], etc. In particular, Baber [2] recently found the Turán density of many 3-uniform hypergraphs using flag algebra methods. For a more complete survey of methods and results on uniform hypergraphs see Peter Keevash's survey paper [30].

A non-uniform hypergraph $H=(V, E)$ consists of a vertex set $V$ and an edge set $E \subseteq$ $2^{V}$. Here the edges of $E$ could have different cardinalities. The set of all the cardinalities of edges in $H$ is denoted by $R(H)$, the set of edge types. For a fixed hypergraph $H$, the Turán density $\pi(H)$ is defined to be $\lim _{n \rightarrow \infty} \max _{G_{n}} h_{n}\left(G_{n}\right)$, where the maximum is taken over all $H$-free hypergraphs $G_{n}$ on $n$ vertices satisfying $R\left(G_{n}\right) \subseteq R(H) . h_{n}\left(G_{n}\right)$, the so called Lubell function, is the expected number of edges in $G_{n}$ hit by a random full chain. The Lubell function has been a very useful tool in extremal poset theory, in particular it has been used in the study of the diamond conjecture.

In Section 2, we show that our notion of $\pi(H)$ is well-defined and is consistent with the usual definition for uniform hypergraphs. We also give examples of Turán densities for several non-uniform hypergraphs. In Section 3, we generalize the supersaturation 
lemma to non-uniform hypergraphs. Then we prove that blowing-up will not affect the Turán density. Using various techniques, we determine the Turán density of every $\{1,2\}$ hypergraph in section 4 . Remarkably, the Turán densities of $\{1,2\}$-hypergraphs are in the set

$$
\left\{1, \frac{9}{8}, \frac{5}{4}\right\} \cup\left\{\frac{3}{2}, \frac{5}{3}, \frac{7}{4}, \ldots, 2-\frac{1}{k}, \ldots\right\} \text {. }
$$

Among $r$-uniform hypergraphs, $r$-partite hypergraphs have the smallest possible Turán density. Erdős proved that any $r$-uniform hypergraph forbidding the complete $r$-uniform $r$-partite hypergraphs can have at most $O\left(n^{r-1 / \delta}\right)$ edges. We generalize this theorem to non-uniform hypergraphs. A hypergraph is degenerate if it has the smallest possible Turán density. For $r$-uniform hypergraphs, a hypergraph $H$ is degenerate if and only if $H$ is the subgraph of a blow-up of a single edge. Unlike the degenerate $r$-uniform hypergraphs, the degenerate non-uniform hypergraphs are not classified. For non-uniform hypergraphs, chains-one natural extension of a single edge-are degenerate. Additionally, every subgraph of a blow-up of a chain is also degenerate. However, we give an example of a degenerate, non-uniform hypergraph not contained in any blow-up of a chain. This leaves open the question of which non-uniform hypergraphs are degenerate.

In Section 6, we consider the suspension of hypergraphs. The suspension of a hypergraph $H$ is a new hypergraph, denoted by $S(H)$, with one additional vertex, *, added to every edge of $H$. In a hypergraph Turán problem workshop hosted by the AIM Research Conference Center in 2011, the following conjecture was posed: $\lim _{t \rightarrow \infty} \pi\left(S^{t}\left(K_{n}^{r}\right)\right)=0$. We conjecture $\lim _{t \rightarrow \infty} \pi\left(S^{t}(H)\right)=|R(H)|-1$ holds for any hypergraph $H$. Some partial results are proved. We will point out the relation between the Turán problems on hypergraphs and extremal poset problems in Section 7.

\section{Non-uniform hypergraphs}

\section{$2.1 \quad$ Notation}

Recall that a hypergraph $H$ is a pair $(V, E)$ with the vertex set $V$ and edge set $E \subseteq 2^{V}$. Here we place no restriction on the cardinalities of edges. The set $R(H):=\{|F|: F \in E\}$ is called the set of its edge types. A hypergraph $H$ is $k$-uniform if $R(H)=\{k\}$. It is non-uniform if it has at least two edge types. For any $k \in R(H)$, the level hypergraph $H^{k}$ is the hypergraph consisting of all edges of cardinality $k$ ( $k$-edges, for short) of $H$. A uniform hypergraph $H$ has only one (non-empty) level graph, i.e., $H$ itself. In general, a non-uniform hypergraph $H$ has $|R(H)|$ (non-empty) level hypergraphs. Throughout the paper, for any finite set $R$ of non-negative integers, we say, $G$ is an $R$-graph if $R(G) \subseteq R$. We write $G_{n}^{R}$ for a hypergraph on $n$ vertices with $R(G) \subseteq R$. We simplify it to $G$ if $n$ and $R$ are clear under context.

Let $R$ be a fixed set of edge types. Let $H$ be an $R$-graph. The number of vertices in $H$ is denoted by $v(H):=|V(H)|$. Our goal is to measure the edge density of $H$ and be able to compare it (in a meaningful way) to the edge density of other $R$ graphs. The 
standard way to measure this density would be:

$$
\mu(H)=\frac{|E(H)|}{\sum_{k \in R(H)}\left(\begin{array}{c}
v(H) \\
k
\end{array}\right)} .
$$

This density ranges from 0 to 1 (as one would expect)-a complete $R$-graph having a density of 1 . Unfortunately, this is no longer a useful measure of density since the number of edges with maximum cardinality will dwarf the number of edges of all other sizes. Specifically, one could take $k$-uniform hypergraph (where $k=\max \{r: r \in R(H)\}$ ) on enough vertices and make its density as close to 1 he likes. The problem is that this $k$-uniform hypergraph is quite different from the complete $R$-graph (when $|R|>1$ ) with the same number of vertices. Instead, we use the Lubell function to measure the edge density. This is adapted from the use of the Lubell function studying families of subsets.

For a non-uniform hypergraph $G$ on $n$ vertices, we define the Lubell function of $G$ as

$$
h_{n}(G):=\sum_{F \in E(G)} \frac{1}{\left(\begin{array}{c}
n \\
|F|
\end{array}\right)}=\sum_{k \in R(G)} \frac{\left|E\left(H^{k}\right)\right|}{\left(\begin{array}{l}
n \\
k
\end{array}\right)} .
$$

The Lubell function is the expected number of edges hit by a random full chain. Namely, pick a uniformly random permutation $\sigma$ on $n$ vertices; define a random full chain $C_{\sigma}$ by

$$
\{\{\emptyset\},\{\sigma(1)\},\{\sigma(1), \sigma(2)\}, \cdots,[n]\} .
$$

Let $X=\left|E(G) \cap C_{\sigma}\right|$, the number of edges hit by the random full chain. Then

$$
h_{n}(G)=\mathrm{E}(X) .
$$

Given two hypergraphs $H_{1}$ and $H_{2}$, we say $H_{1}$ is a subgraph of $H_{2}$, denoted by $H_{1} \subseteq$ $H_{2}$, if there exists a 1-1 map $f: V\left(H_{1}\right) \rightarrow V\left(H_{2}\right)$ so that $f(F) \in E\left(H_{2}\right)$ for any $F \in$ $E\left(H_{1}\right)$. Whenever this occurs, we say the image $f\left(H_{1}\right)$ is an ordered copy of $H_{2}$, written as $H_{1} \stackrel{f}{\hookrightarrow} H_{2}$. A necessary condition for $H_{1} \subseteq H_{2}$ is $R\left(H_{1}\right) \subseteq R\left(H_{2}\right)$. Given a subset $K \subseteq V(H)$ and a subset $S \subseteq R(H)$, the induced subgraph, denoted by $H^{[S]}[K]$, is a hypergraph on $K$ with the edge set $\{F \in E(H): F \subseteq K$ and $|F| \in S\}$. When $S=R(H)$, we simply write $H[K]$ for $H^{[S]}[K]$.

Given a positive integer $n$ and a subset $R \subseteq[n]$, the complete hypergraph $K_{n}^{R}$ is a hypergraph on $n$ vertices with edge set $\bigcup_{i \in R}\left(\begin{array}{c}{[n]} \\ i\end{array}\right)$. For example, $K_{n}^{\{k\}}$ is the complete $k$-uniform hypergraph. $K_{n}^{[k]}$ is the non-uniform hypergraph with all possible edges of cardinality at most $k$. 


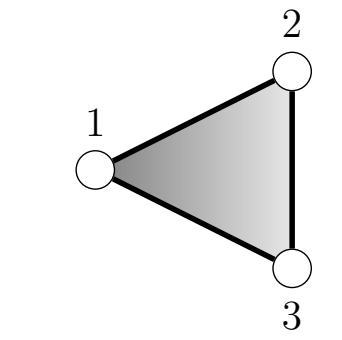

Illustration of $K_{3}^{\{2,3\}}$

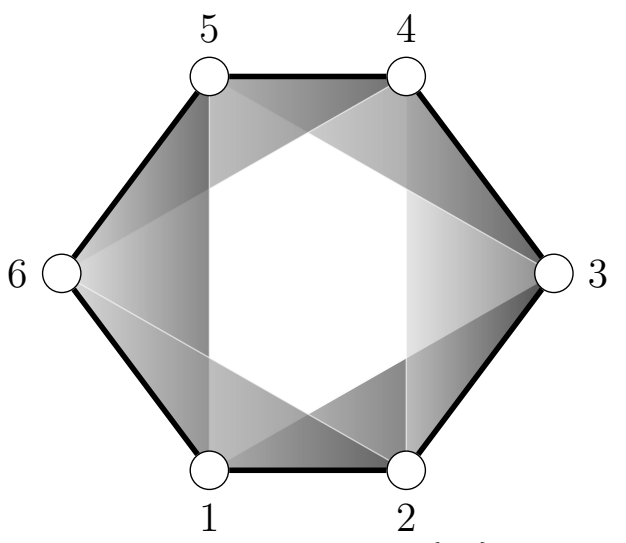

A (tight) cycle $C_{6}^{\{2,3\}}$

Given a family of hypergraphs $\mathcal{H}$ with common set of edge-types $R$, we define $\pi_{n}(\mathcal{H}):=\max \left\{h_{n}(G): v(G)=n, G \subseteq K_{n}^{R}\right.$, and $G$ contains no subgraph in $\left.\mathcal{H}\right\}$.

A hypergraph $G:=G_{n}^{R}$ is extremal with respect to the family $\mathcal{H}$ if

1. $G$ contains no subgraph in $\mathcal{H}$.

2. $h_{n}(G)=\pi_{n}(\mathcal{H})$.

The Turán density of $\mathcal{H}$ is defined to be

$$
\begin{aligned}
\pi(\mathcal{H}): & =\lim _{n \rightarrow \infty} \pi_{n}(\mathcal{H}) \\
& =\lim _{n \rightarrow \infty} \max \left\{\sum_{F \in E(G)} \frac{1}{\left(\begin{array}{c}
n \\
|F|
\end{array}\right)}: v(G)=n, G \subseteq K_{n}^{R} \text { contains no subgraph in } \mathcal{H}\right\}
\end{aligned}
$$

when the limit exists; we will soon show that this limit always exists. When $\mathcal{H}$ contains one hypergraph $H$, then we write $\pi(H)$ instead of $\pi(\{H\})$.

Throughout, we will consider $n$ growing to infinity, and $R$ to be a fixed set (not growing with $n$ ). Note that $\pi(\mathcal{H})$ agrees with the usual definition of

$$
\pi(\mathcal{H})=\lim _{n \rightarrow \infty} \frac{\operatorname{ex}(\mathcal{H}, n)}{\left(\begin{array}{l}
n \\
k
\end{array}\right)}
$$

when $\mathcal{H}$ is a set of $k$-uniform hypergraphs. The following result is a direct generalization Katona-Nemetz-Simonovit's theorem [28].

Theorem 1. For any family $\mathcal{H}$ of $R$-graphs, $\pi(\mathcal{H})$ is well-defined, i.e. the limit $\lim _{n \rightarrow \infty} \pi_{n}(\mathcal{H})$ exists. 
Proof. It suffices to show that $\pi_{n}(\mathcal{H})$, viewed as a sequence in $n$, is decreasing.

Write $R=\left\{k_{1}, k_{2}, \ldots, k_{r}\right\}$. Let $G_{n} \subseteq K_{n}^{R}$ be a hypergraph with $v\left(G_{n}\right)=n$ not containing $\mathcal{H}$ and with Lubell value $h_{n}\left(G_{n}\right)=\pi_{n}(\mathcal{H})$. For any $\ell<n$, consider a random subset $S$ of the vertices of $G$ with size $|S|=\ell$.

Let $G_{n}[S]$ be the induced subgraph of $G_{n}$ (whose vertex set is restricted to $S$ ). Clearly

$$
\pi_{\ell}(\mathcal{H}) \geqslant \mathbb{E}\left(h_{\ell}\left(G_{n}[S]\right)\right) .
$$

Write $E\left(G_{n}\right)=E_{k_{1}} \cup E_{k_{2}} \cup \ldots \cup E_{k_{r}}$ where $E_{k_{i}}$ contains all the edges of size $k_{i}$. Note that the expected number of edges of size $k_{i}$ in $G_{n}[S]$ is precisely $\frac{\left(\begin{array}{l}\ell \\ k_{i}\end{array}\right)}{\left(\begin{array}{c}n \\ k_{i}\end{array}\right)}\left|E_{k_{i}}\right|$. It follows that

$$
\begin{aligned}
\pi_{\ell}(\mathcal{H}) & \geqslant \mathbb{E}\left(h_{\ell}\left(G_{n}[S]\right)\right) \\
& =\sum_{i=1}^{r} \frac{\mathbb{E}\left(\left|E_{k_{i}} \cap\left(\begin{array}{c}
S \\
k_{i}
\end{array}\right)\right|\right)}{\left(\begin{array}{c}
\ell \\
k_{i}
\end{array}\right)} \\
& =\sum_{i=1}^{r} \frac{\frac{\left(\begin{array}{l}
\ell \\
k_{i}
\end{array}\right)}{\left(\begin{array}{c}
n \\
k_{i}
\end{array}\right)}\left|E_{k_{i}}\right|}{\left(\begin{array}{c}
\ell \\
k_{i}
\end{array}\right)} \\
& =\sum_{i=1}^{r} \frac{\left|E_{k_{i}}\right|}{\left(\begin{array}{c}
n \\
k_{i}
\end{array}\right)} \\
& =h_{n}\left(G_{n}\right) \\
& =\pi_{n}(\mathcal{H}) .
\end{aligned}
$$

The sequence $\pi_{n}(\mathcal{H})$ is non-negative and decreasing; therefore it converges.

For a fixed set $R:=\left\{k_{1}, k_{2}, \ldots, k_{r}\right\}$ (with $k_{1}<k_{2}<\cdots<k_{r}$ ), an $R$-flag is an $R$-graph containing exactly one edge of each size. The chain $C^{R}$ is a special $R$-flag with the edge set

$$
\mathrm{E}\left(C^{R}\right)=\left\{\left[k_{1}\right],\left[k_{2}\right], \ldots,\left[k_{r}\right]\right\}
$$

Proposition 2. For any hypergraph $H$, the following statements hold.

1. $|R(H)|-1 \leqslant \pi_{n}(H) \leqslant|R(H)|$.

2. For subgraph $H^{\prime}$ of $H$, we have $\pi\left(H^{\prime}\right) \leqslant \pi(H)-|R(H)|+\left|R\left(H^{\prime}\right)\right|$.

3. For any $R$-flag $L$ on $m$ vertices and any $n \geqslant m$, we have $\pi_{n}(L)=|R|-1$.

Proof. Pick any maximal proper subset $R^{\prime}$ of $R(H)$. Consider the complete graph $K_{n}^{R^{\prime}}$. Since $K_{n}^{R^{\prime}}$ misses one type of edge in $R(H) \backslash R^{\prime}$, it does not contain $H$ as a subgraph. Thus

$$
\pi_{n}(H) \geqslant h_{n}\left(K_{n}^{R^{\prime}}\right)=\left|R^{\prime}\right|=|R(H)|-1 .
$$

The upper bound is due to the fact $h_{n}\left(K_{n}^{R(H)}\right)=|R(H)|$. 
Proof of item 2 is similar. Let $S=R\left(H^{\prime}\right)$ and $G_{n}^{S}$ be an extremal hypergraph for $\pi_{n}\left(H^{\prime}\right)$. Extend $G_{n}^{S}$ to $G_{n}^{R(H)}$ by adding all the edges with cardinalities in $R(H) \backslash S$. The resulting graph $G_{n}^{R(H)}$ is $H$-free. We have

$$
\pi_{n}(H) \geqslant \pi_{n}\left(G_{n}^{R(H)}\right)=\pi_{n}\left(G_{n}^{S}\right)+|R(H)|-|S|=|R(H)|-\left|R\left(H^{\prime}\right)\right|+\pi_{n}\left(H^{\prime}\right) .
$$

Taking the limit as $n$ goes to infinity, we have

$$
\pi(H) \geqslant|R(H)|-\left|R\left(H^{\prime}\right)\right|+\pi\left(H^{\prime}\right) .
$$

Finally, for item 3, consider $L$-free hypergraph $G_{n}^{R}$. Pick a random $n$-permutation $\sigma$ uniformly. Let $X$ be the number of edges of $G_{n}^{R}$ hit by a random flag $\sigma(L)$. Note that each edge $F$ has probability $\frac{1}{\left(\begin{array}{c}n \\ |F|\end{array}\right)}$ of being hit by $\sigma(L)$. We have

$$
\mathrm{E}(X)=\sum_{F \in E(G)} \frac{1}{\left(\begin{array}{c}
n \\
|F|
\end{array}\right)}=h_{n}(G) .
$$

Since $G_{n}^{R}$ is $L$-free, we have $X \leqslant r-1$. Taking the expectation, we have

$$
h_{n}\left(G_{n}^{R}\right)=\mathrm{E}(X) \leqslant r-1 .
$$

Hence, $\pi_{n}(H) \leqslant r-1$. The result is followed after combining with item 1 .

Definition 3. A hypergraph $H$ is called degenerate if $\pi(H)=|R(H)|-1$.

By Proposition 2, flags, and specifically chains, are degenerate hypergraphs. A necessary condition for $H$ to be degenerate is that every level hypergraph $H^{k_{i}}$ is $k_{i}$-partite. The following examples will show that the converse is not true.

Example 1: The complete hypergraph $K_{2}^{\{1,2\}}$ has three edges $\{1\},\{2\}$, and $\{1,2\}$. We claim

$$
\pi\left(K_{2}^{\{1,2\}}\right)=\frac{5}{4}
$$

The lower bound is from the following construction. Partition $[n]$ into two parts $A$ and $B$ of nearly equal size. Consider the hypergraph $G$ with the edge set

$$
E(G)=\left(\begin{array}{c}
A \\
1
\end{array}\right) \cup\left(\left(\begin{array}{c}
{[n]} \\
2
\end{array}\right) \backslash\left(\begin{array}{c}
A \\
2
\end{array}\right)\right)
$$

It is easy to check $h_{n}(G)=\frac{5}{4}+O\left(\frac{1}{n}\right)$ and that $G$ contains no copy of $K_{2}^{\{1,2\}}$.

Now we prove the upper bound. Consider any $K_{2}^{\{1,2\}}$-free hypergraph $G$ of edge-type $\{1,2\}$ on $n$ vertices. Let $A$ be the set of all singleton edges. For any $x, y \in A, x y$ is not a 2-edge of $G$. We have

$$
h_{n}(G) \leqslant \frac{|A|}{n}+1-\frac{\left(\begin{array}{c}
|A| \\
2
\end{array}\right)}{\left(\begin{array}{c}
n \\
2
\end{array}\right)}
$$




$$
\begin{aligned}
& =1+\frac{|A|}{n}-\frac{|A|^{2}}{n^{2}}+O\left(\frac{1}{n}\right) \\
& \leqslant 1+\frac{1}{4}+O\left(\frac{1}{n}\right) .
\end{aligned}
$$

In the last step, we use the fact that $f(x)=1+x-x^{2}$ has the maximum value $\frac{5}{4}$. Combining the upper and lower bounds we have $\pi\left(K_{2}^{\{1,2\}}\right)=\frac{5}{4}$.

The argument is easily generalized to the complete graph $K_{k}^{\{1, k\}}$ (for $k>1$ ). We have

$$
\pi\left(K_{k}^{1, k}\right)=1+\frac{k-1}{k^{\frac{k}{k-1}}} .
$$

Definition 4. The suspension of a hypergraph $H$, denoted $S(H)$, is the hypergraph with $V=V(H) \bigcup\{*\}$ where $\{*\}$ is an element not in $V(H)$, and edge set $E=\{F \bigcup\{*\}: F \in E(H)\}$. We write $S^{t}(H)$ to denote the hypergraph obtained by iterating the suspension operation $t$-times, i.e. $S^{2}(H)=S(S(H))$ and $S^{3}(H)=S(S(S(H)))$, etc.

For a family $\mathcal{H}$ of hypegraphs, let $S(\mathcal{H})=\{S(H): H \in \mathcal{H}\}$ and $S^{t}(\mathcal{H})=\left\{S^{t}(H): H \in\right.$ $\mathcal{H}\}$ for $t \geqslant 1$.

Definition 5. Let $H$ be a hypergraph. The $k$-degree of a vertex $x$, denoted $d_{k}(x)$, is the number of edges of size $k$ containing $x$.

Example 2: Consider $H:=S\left(K_{2}^{\{1,2\}}\right)$. The edges of $H$ are $\{1,2\},\{2,3\},\{1,2,3\}$. We claim

$$
\pi\left(S\left(K_{2}^{\{1,2\}}\right)\right)=\frac{5}{4}
$$

The lower bound is from the following construction. Partition $[n]$ into two parts $A$ and $B$ of nearly equal size. Consider the hypergraph $G$ with the edge set $E=E_{2} \cup E_{3}$ where $E_{2}=\left(\begin{array}{c}A \\ 2\end{array}\right) \bigcup\left(\begin{array}{c}B \\ 2\end{array}\right)$ and $E_{3}=\left(\begin{array}{c}{[n]} \\ 3\end{array}\right) \backslash\left(\left(\begin{array}{c}A \\ 3\end{array}\right) \bigcup\left(\begin{array}{c}B \\ 3\end{array}\right)\right)$. It is easy to check $h_{n}(G)=\frac{5}{4}+O\left(\frac{1}{n}\right)$ and that $G$ is $H$-free.

Now we prove the upper bound. Consider any $H$-free hypergraph $G$ of edge-type $\{2,3\}$ on $n$ vertices. Recall that $d_{2}(v)$ denotes the number of 2-edges that contain $v$. For each pair of 2-edges that intersect $v$ there is a unique 3 -set containing those two pairs. This 3set cannot appear in the edge set of $G$ since $G$ is $H$-free. We say that the edge is forbidden. Note that each 3-edge may be forbidden up to 3 times in this manner-depending on which of the three vertices we call $v$. Hence there are at least $\frac{1}{3} \sum_{v \in[n]}\left(\begin{array}{c}d_{2}(v) \\ 2\end{array}\right)$ 3-edges which are not in $G$. Note that this is true for any $H$-free graph $G$ with number of vertices. Hence

$$
\begin{aligned}
h_{n}(G) & \leqslant \frac{\left(\begin{array}{l}
n \\
3
\end{array}\right)-\frac{1}{3} \sum_{v \in[n]}\left(\begin{array}{c}
d_{2}(v) \\
2
\end{array}\right)}{\left(\begin{array}{l}
n \\
3
\end{array}\right)}+\frac{\frac{1}{2} \sum_{v \in[n]} d_{2}(v)}{\left(\begin{array}{c}
n \\
2
\end{array}\right)} \\
& =1-\frac{\sum_{v \in[n]} d_{2}(v)^{2}}{6\left(\begin{array}{l}
n \\
3
\end{array}\right)}+\left(\frac{1}{6\left(\begin{array}{c}
n \\
3
\end{array}\right)}+\frac{1}{2\left(\begin{array}{c}
n \\
2
\end{array}\right)}\right) \sum_{v \in[n]} d_{2}(v) .
\end{aligned}
$$


Applying Cauchy-Schwarz Inequality and letting $m:=\sum_{v} d_{2}(v)$, we have

$$
\begin{aligned}
h_{n}(G) & \leqslant \frac{-\left(\sum_{v \in[n]} d_{2}(v)\right)^{2}}{6 n\left(\begin{array}{l}
n \\
3
\end{array}\right)}+\left(\frac{1}{6\left(\begin{array}{c}
n \\
3
\end{array}\right)}+\frac{1}{2\left(\begin{array}{c}
n \\
2
\end{array}\right)}\right) \sum_{v \in[n]} d_{2}(v)+1 \\
& =-\frac{m^{2}}{n^{4}}+\frac{m}{n^{2}}+1+O\left(\frac{1}{n}\right) \\
& \leqslant \frac{5}{4}+O\left(\frac{1}{n}\right) .
\end{aligned}
$$

In the last step, we use the fact that $f(x)=1+x-x^{2}$ has the maximum value $\frac{5}{4}$. Taking the limit, we get $\pi\left(S\left(K_{2}^{\{1,2\}}\right)\right) \leqslant \frac{5}{4}$.

We can generalize this construction to the following lower bound for $S^{k}\left(K_{2}^{\{1,2\}}\right)$ (the $k$-th suspension of $\left.K_{2}^{\{1,2\}}\right)$. Partition $V$ into to subsets $A$ and $B$ of size $x n$ and $(1-x) n$ respectively. Let $H$ be a $\{k+1, k+2\}$-hypergraph with the edge set:

$$
\left(\begin{array}{c}
A \\
k+1
\end{array}\right) \cup\left(\begin{array}{c}
B \\
k+1
\end{array}\right) \cup\left[\left(\begin{array}{c}
V \\
k+2
\end{array}\right) \backslash\left(\begin{array}{c}
A \\
k+2
\end{array}\right) \backslash\left(\begin{array}{c}
B \\
k+2
\end{array}\right)\right] .
$$

It is clear that $H$ contains no subhypergraph $S^{k}\left(K_{2}^{\{1,2\}}\right)$. Thus, for any $x \in(0,1)$,

$$
\pi\left(S^{k}\left(K_{2}^{\{1,2\}}\right)\right) \geqslant 1+x^{k+1}-x^{k+2}+(1-x)^{k+1}-(1-x)^{k+2} .
$$

Conjecture 6. For any $k \geqslant 2, \pi\left(S^{k}\left(K_{2}^{\{1,2\}}\right)\right)=1+\max _{x \in(0,1)}\left\{x^{k+1}-x^{k+2}+(1-x)^{k+1}-\right.$ $\left.(1-x)^{k+2}\right\}$.

\section{Supersaturation and Blowing-up}

There is a natural generalization of the supersaturation lemma and blowing-up in nonuniform hypergraphs.

Lemma 7 (Supersaturation of Families). Let $\mathcal{H}$ be a finite family of $R$-graphs. For any constant $a>0$ there exist positive constants $b$ and $n_{0}$ so that if $G$ is an $R$-graph on $n>n_{0}$ vertices with $h_{n}(G)>\pi(\mathcal{H})+$ a then $G$ contains at least $b\left(\begin{array}{c}n \\ v(H)\end{array}\right)$ copies of some $H \in \mathcal{H}$.

Proof. Let $r:=|R(\mathcal{H})|$ and let $R=R(\mathcal{H})$. Add independent vertices to members of $\mathcal{H}$ so that every graph in $\mathcal{H}$ has the same number of vertices. Note that adding these vertices does not change the Turán density of $H$. Since $\lim _{n \rightarrow \infty} \pi_{n}(\mathcal{H})=\pi(\mathcal{H})$, there exists an $n_{0}>0$ so that if $m>n_{0}$ then $\pi_{m}(\mathcal{H})<\pi(\mathcal{H})+\frac{a}{2}$. Suppose that $n_{0}<m<n$ and $G$ is an $R$-graph on $n$ vertices with $h_{n}(G)>\pi(\mathcal{H})+a$. Then, $G$ must contain $\frac{a}{2 r}\left(\begin{array}{l}n \\ m\end{array}\right) m$-sets $M \subset V(G)$ satisfying $h_{m}(G[M])>\pi(\mathcal{H})+\frac{a}{2}$. Otherwise we have

$$
\sum_{M} h_{m}(G[M]) \leqslant\left(\pi(\mathcal{H})+\frac{a}{2}\right)\left(\begin{array}{c}
n \\
m
\end{array}\right)+\frac{a}{2 r}\left(\begin{array}{c}
n \\
m
\end{array}\right) r=(\pi(\mathcal{H})+a)\left(\begin{array}{c}
n \\
m
\end{array}\right) .
$$


And we have that

$$
\begin{aligned}
\sum_{M} h_{m}(G[M]) & =\sum_{M} \sum_{\substack{F \in E(G) \\
F \subseteq M}} \frac{1}{\left(\begin{array}{c}
m \\
|F|
\end{array}\right)} \\
& =\sum_{F \in E(G)} \sum_{M \supseteq F} \frac{1}{\left(\begin{array}{c}
m \\
|F|
\end{array}\right)} \\
& =\sum_{F \in E(G)} \frac{\left(\begin{array}{c}
n-|F| \\
m-|F|
\end{array}\right)}{\left(\begin{array}{c}
m \\
|F|
\end{array}\right)} \\
& =\sum_{F \in E(G)} \frac{\left(\begin{array}{c}
n \\
m
\end{array}\right)}{\left(\begin{array}{c}
n \\
|F|
\end{array}\right)} \\
& =\left(\begin{array}{c}
n \\
m
\end{array}\right) h_{n}(G) .
\end{aligned}
$$

But this implies $h_{n}(G) \leqslant \pi(\mathcal{H})+a$ which is contrary to the assumption that $h_{n}(G)>$ $\pi(\mathcal{H})+a$.

Since $m>n_{0}$ it follows that each of the $\frac{a}{2 r}\left(\begin{array}{l}n \\ m\end{array}\right) m$-sets $M \subset V(G)$ satisfying $h_{m}(G[M])>\pi(\mathcal{H})+\frac{a}{2}$ contains some member of $\mathcal{H}$. Let $v=v(H)=|V(H)|$ for each $H \in \mathcal{H}$. Recall that we added vertices to members of $\mathcal{H}$ as necessary so that every graph had the same number of vertices. Then, counted by multiplicity, there are at least $\frac{a}{2 r}\left(\begin{array}{l}n \\ m\end{array}\right) /\left(\begin{array}{c}n-v \\ m-v\end{array}\right)=\frac{a}{2 r}\left(\begin{array}{c}m \\ v\end{array}\right)^{-1}\left(\begin{array}{l}n \\ v\end{array}\right)$ members of $\mathcal{H}$ in $G$. By the pigeon hole principle, at least one member of $\mathcal{H}$ appears in $G$ at least $b\left(\begin{array}{l}n \\ v\end{array}\right)$ times where $b=\frac{a}{2 r|\mathcal{H}|}\left(\begin{array}{c}m \\ v\end{array}\right)^{-1}$.

Supersaturation can be used to show that "blowing up" does not change the Turán density $\pi(H)$ just like in the uniform cases.

Definition 8. For any hypergraph $H_{n}$ and positive integers $s_{1}, s_{2}, \ldots, s_{n}$, the blow-up of $H$ is a new hypergraph $(V, E)$, denoted by $H_{n}\left(s_{1}, s_{2}, \ldots, s_{n}\right)$, satisfying

1. $V:=\sqcup_{i=1}^{n} V_{i}$, where $\left|V_{i}\right|=s_{i}$.

2. $E=\cup_{F \in \mathrm{E}(H)} \prod_{i \in F} V_{i}$.

When $s_{1}=s_{2}=\cdots=s_{n}=s$, we simply write it as $H(s)$. The blow-up of a family $\mathcal{H}$ of finitely many non-uniform hypergraphs is defined as $\mathcal{H}(s)=\{H(s): H \in \mathcal{H}\}$.

Consider the following simple example. Take $H$ to be the hypergraph with vertex set [3] and edge set $E=\{\{1,2\},\{1,2,3\}\}$, a chain. Consider the blow-ups $H(2,1,1)$ and $H(1,1,2)$ illustrated below. 


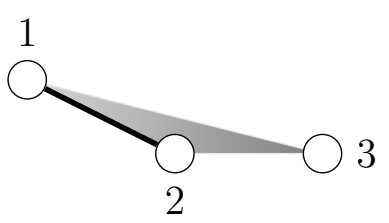

$H$

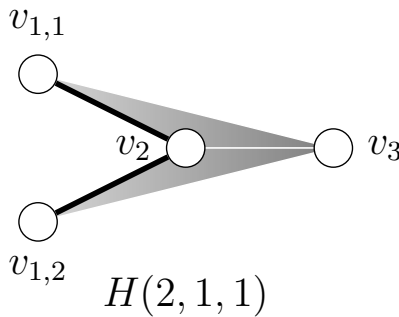

$H(2,1,1)$

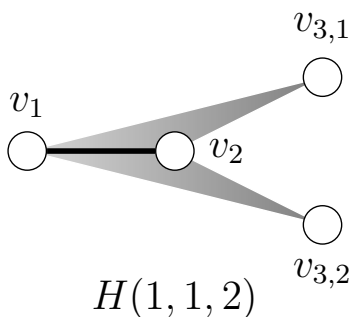

In the blow-up $H(2,1,1)$ vertex 1 splits into vertices $v_{1,1}$ and $v_{1,2}$; vertex 2 becomes $v_{2}$ and vertex 3 becomes $v_{3}$. In the blow-up $H(1,1,2)$ vertex 3 splits into vertices $v_{3,1}$ and $v_{3,2}$; vertex 1 becomes $v_{1}$ and vertex 2 becomes $v_{2}$.

Theorem 9 (Blow-ups of Families). Let $\mathcal{H}$ be a finite family of hypergraphs and let $s \geqslant 2$. Then $\pi(\mathcal{H}(s))=\pi(\mathcal{H})$.

Proof. First, it is clear that $\pi(\mathcal{H}) \leqslant \pi(\mathcal{H}(s))$ since any $\mathcal{H}$-free graph $G$ is also $\mathcal{H}(s)$-free. We will now show that for any $\epsilon>0$ that $\pi(\mathcal{H}(s))<\pi(\mathcal{H})+\epsilon$.

Let $\epsilon>0$. Let $R=R(\mathcal{H})$. Again, add independent vertices as necessary to the graphs in $\mathcal{H}$ so that they all have the same number of vertices. Let $v$ denote the common size of the vertex set of every element in $\mathcal{H}$. By the supersaturation lemma, there exists an $n_{0}$ and a $b>0$ so that if $G$ is an $R$-graph on $n>n_{0}$ vertices, then $G$ contains at least $b\left(\begin{array}{l}n \\ v\end{array}\right)$ copies of some $H \in \mathcal{H}$. We will show that $H(s)$ is contained in $G$.

Consider an auxiliary $v$-uniform hypergraph $U$ with $V(U)=V(G)$. A $v$-set of the vertices forms an edge in $U$ if and only if the corresponding $v$-set in $G$ is a copy of $H$. Note that $U$ contains at least $b\left(\begin{array}{l}n \\ v\end{array}\right)$ edges. For $n$ large enough, and any $S>1$ it follows that $U$ contains a copy of $K=K_{v}^{v}(S)$. This follows since $K$ is $v$-uniform and $v$-partite implying that $\pi(K)=0$ and $h_{n}(U) \geqslant b>0$. Fix one such $K$ in $U$. Color each of the edges of $K$ with one of the $v$ ! colors corresponding to the possible orderings with which the vertices of $H$ are mapped into the parts of $K$. By the pigeon hole principle, one of the color classes contains at least $S^{v} / v$ ! edges. For large enough $S$ (such that $S^{v} / v ! \geqslant s$ ) it follows that $U$ contains a monochromatic copy of $K_{v}^{v}(s)$. This monochromatic copy of $K_{v}^{v}(s)$ in $U$ corresponds to a copy of $H(s)$ in $G$. Hence $\pi(\mathcal{H}(s))<\pi(\mathcal{H})+\epsilon$ as desired.

Corollary 10 (Squeeze Theorem). Let $H$ be any hypergraph. If there exists a hypergraph $H^{\prime}$ and integer $s \geqslant 2$ such that $H^{\prime} \subseteq H \subseteq H^{\prime}(s)$ then $\pi(H)=\pi\left(H^{\prime}\right)$.

Proof. One needs only observe that for any hypergraphs $H_{1} \subseteq H_{2} \subseteq H_{3}$ that $\pi\left(H_{1}\right) \leqslant$ $\pi\left(H_{2}\right) \leqslant \pi\left(H_{3}\right)$. If $H_{3}=H_{1}(s)$ for some $s \geqslant 2$ then $\pi\left(H_{1}\right)=\pi\left(H_{3}\right)$ by the previous theorem.

\section{Turán Densities of $\{1,2\}$-hypergraphs}

In this section we will determine the Turán density for any hypergraph $H$ with $R(H)=$ $\{1,2\}$. We begin with the following more general result. 
Theorem 11. Let $H=H^{1} \cup H^{k}$ be a hypergraph with $R(H)=\{1, k\}$ and $E\left(H^{1}\right)=$ $V\left(H^{k}\right)$; i.e., each vertex of $H^{k}$ is an edge of $H^{1}$. Then

$$
\pi(H)= \begin{cases}1+\pi\left(H^{k}\right) & \text { if } \pi\left(H^{k}\right) \geqslant 1-\frac{1}{k} \\ 1+\left(\frac{1}{k\left(1-\pi\left(H^{k}\right)\right)}\right)^{1 /(k-1)}\left(1-\frac{1}{k}\right) & \text { otherwise. }\end{cases}
$$

Proof. For each $n \in \mathbb{N}$, let $G_{n}$ be any $H$-free graph on $n$ vertices with $h_{n}\left(G_{n}\right)=\pi_{n}(H)$. Partition the vertices of $G_{n}$ into $X_{n}=\left\{v \in V\left(G_{n}\right):\{v\} \in E(G)\right\}$ and $\bar{X}_{n}$ containing everything else. Say that $\left|X_{n}\right|=x_{n} n$ and $\left|\bar{X}_{n}\right|=\left(1-x_{n}\right) n$ for some $x_{n} \in[0,1]$. Since $\left(x_{n}\right)$ is a sequence in $[0,1]$ it has a convergent subsequence. Consider $\left(x_{n}\right)$ to be the convergent subsequence, and say that $x_{n} \rightarrow x \in[0,1]$. With the benefit of hindsight, we know that $x>0$, however, for the upper bound portion of this proof we will not assume this knowledge.

Since there is no copy of $H$ in $G_{n}$, it follows that $G_{n}\left[X_{n}\right]$ contains no copy of $H^{k}$. We have that

$$
\begin{aligned}
\pi(H) & =\lim _{n \rightarrow \infty} h_{n}\left(G_{n}\right) \\
& =\lim _{n \rightarrow \infty} \sum_{F \in G^{1}} \frac{1}{\left(\begin{array}{l}
n \\
1
\end{array}\right)}+\sum_{F \in G^{k}} \frac{1}{\left(\begin{array}{l}
n \\
k
\end{array}\right)} \\
& \leqslant \lim _{n \rightarrow \infty} \frac{x_{n} n}{\left(\begin{array}{l}
n \\
1
\end{array}\right)}+\frac{\left(\begin{array}{l}
n \\
k
\end{array}\right)-\left(1-\pi_{x_{n} n}\left(H^{k}\right)\right)\left(\begin{array}{c}
x_{n} n \\
k
\end{array}\right)}{\left(\begin{array}{l}
n \\
k
\end{array}\right)} \\
& =\lim _{n \rightarrow \infty} 1+x_{n}-\left(1-\pi_{x_{n} n}\left(H^{k}\right)\right) \frac{\left(\begin{array}{c}
x_{n} n \\
k
\end{array}\right)}{\left(\begin{array}{l}
n \\
k
\end{array}\right)} \\
& \leqslant \lim _{n \rightarrow \infty} \begin{cases}1+\frac{1}{\sqrt{n}} & \text { if } x_{n} n \leqslant \sqrt{n} \\
1+x_{n}-\left(1-\pi_{x_{n} n}\left(H^{k}\right)\right) x_{n}^{k} & \text { if } x_{n} n>\sqrt{n}, \\
& \leqslant \max \left\{1,1+x-\left(1-\pi\left(H^{k}\right)\right) x^{k}\right\} .\end{cases}
\end{aligned}
$$

Let $f(x)=1+x-\left(1-\pi\left(H^{k}\right)\right) x^{k}$ and then note that

$$
\pi(H)=\lim _{n \rightarrow \infty} h_{n}(G) \leqslant \max _{x \in[0,1]} f(x) .
$$

An easy calculus exercise shows that $f^{\prime \prime}(x)<0$ for all $x>0$, and $f^{\prime}(x)=0$ when $x=\left(\frac{1}{k\left(1-\pi\left(H^{k}\right)\right)}\right)^{\frac{1}{k-1}}$. If $\frac{1}{k\left(1-\pi\left(H^{k}\right)\right)} \geqslant 1$ then $f^{\prime}(x)>0$ when $x \in[0,1)$ and hence $f(x)$ is maximized when $x=1$. Note that $f(1)=1+\pi\left(H^{k}\right)$. If, on the other hand, $\frac{1}{k\left(1-\pi\left(H^{k}\right)\right)}<1$ it follows that $f(x)$ is maximized at $x=\left(\frac{1}{k\left(1-\pi\left(H^{k}\right)\right)}\right)^{1 /(k-1)}$. Together, this gives us

$$
\pi(H) \leqslant \begin{cases}1+\pi\left(H^{k}\right) & \text { if } \pi\left(H^{k}\right) \geqslant 1-\frac{1}{k} \\ 1+\left(\frac{1}{k\left(1-\pi\left(H^{k}\right)\right)}\right)^{1 /(k-1)}\left(1-\frac{1}{k}\right) & \text { otherwise. }\end{cases}
$$


To get equality, take $x$ that maximizes $f(x)$ as above. For any $n \in \mathbb{N}$ (thinking of $n \rightarrow \infty)$ partition $[n]$ into two sets $X$ and $\bar{X}$ with $|X|=x n$ and $|\bar{X}|=(1-x) n$. Let $E\left(G^{1}\right)=\{\{v\}: v \in X\}$ and let $g^{k}$ be a $k$-uniform graph on $x n$ vertices attaining $\left|E\left(g^{k}\right)\right|=\operatorname{ex}\left(x n, H^{k}\right)$ and $g^{k}$ is $H^{k}$-free. Then

$$
E\left(G^{k}\right)=\left\{F \in\left(\begin{array}{c}
{[n]} \\
k
\end{array}\right) \text { : either } F \in E\left(g^{k}\right) \text { or } F \cap \bar{X} \neq \emptyset\right\} .
$$

Then $G=G^{1} \cup G^{k}$ is $H$-free and (by choice of $x$ ) we have that $\lim _{n \rightarrow \infty} h_{n}(G)$ attains the upper bound of $\pi(H)$.

Let us now return to the task of determining $\pi(H)$ when $H=H^{1} \cup H^{2}$.

Proposition 12. Let $H=H^{1} \cup H^{2}$. If $H^{2}$ is not bipartite, then

$$
\pi(H)=1+\pi\left(H^{2}\right)=1+\left(1-\frac{1}{\chi\left(H^{2}\right)-1}\right)=2-\frac{1}{\chi\left(H^{2}\right)-1} .
$$

Proof. First, $\pi(H) \geqslant 1+\pi\left(H^{2}\right)$ since one can construct an $H$-free graph $G_{n}$ by letting

$$
E\left(G_{n}\right)=\left\{\{v\}: v \in V\left(G_{n}\right)\right\} \cup E\left(G_{n}^{\prime}\right)
$$

where $G_{n}^{\prime}$ attains $h_{n}\left(G_{n}^{\prime}\right)=\pi_{n}\left(H^{2}\right)$ and $G_{n}^{\prime}$ is $H^{2}$-free. Then

$$
\pi(H) \geqslant \lim _{n \rightarrow \infty} h_{n}\left(G_{n}\right)=\lim _{n \rightarrow \infty} 1+\pi_{n}\left(H^{2}\right)=1+\pi\left(H^{2}\right) .
$$

To get the upper-bound, first add every missing 1-edge into $H$, call the new graph $H^{\prime}$. Note that $\pi(H) \leqslant \pi\left(H^{\prime}\right)$. Note that we didn't change the edge set $H^{2}$. The Erdős-StoneSimonovits theorem states that if $H^{2}$ is not bipartite, then $\pi\left(H^{2}\right)=1-\frac{1}{\chi\left(H^{2}\right)-1}$. Also, if $H^{2}$ is not bipartite, then $\chi\left(H^{2}\right) \geqslant 3$. With the added vertices, taking $k=2$, we apply the previous theorem. Since

$$
\pi\left(H^{2}\right)=1-\frac{1}{\chi\left(H^{2}\right)-1} \geqslant 1-\frac{1}{2}
$$

we may conclude that $\pi(H) \leqslant \pi\left(H^{\prime}\right)=1+\pi\left(H^{2}\right)$.

It remains to investigate the cases when $H^{2}$ is bipartite.

Proposition 13. Let $H=H^{1} \cup H^{2}$. If $H^{2}$ is bipartite and $K_{2}^{\{1,2\}} \subseteq H$ then $\pi(H)=\frac{5}{4}$.

Proof. First, in example 1, we computed $\pi\left(K_{2}^{\{1,2\}}\right)=\frac{5}{4}$. Second, $H$ must be contained in some blow-up of $K_{2}^{\{1,2\}}$ since $H^{2}$ is bipartite, i.e. there exists some $s>2$ such that $H \subseteq K_{2}^{\{1,2\}}(s)$. So, by the squeeze theorem we have

$$
\frac{5}{4}=\pi\left(K_{2}^{\{1,2\}}\right) \leqslant \pi(H) \leqslant \pi\left(K_{2}^{\{1,2\}}(s)\right)=\frac{5}{4} .
$$

Hence $\pi(H)=\frac{5}{4}$ as claimed. 
Definition 14. We will say that $H=H^{1} \cup H^{2}$ is a closed path (from $x_{1}$ to $x_{k}$ ) of length $k$ if $V(H)=\left\{x_{1}, x_{2}, \ldots, x_{k}\right\}$ and $E\left(H^{1}\right)=\left\{\left\{x_{1}\right\},\left\{x_{k}\right\}\right\}$ and $E\left(H^{2}\right)=\left\{\left\{x_{i}, x_{i+1}\right\}: 1 \leqslant\right.$ $i \leqslant k-1\}$. We will denote a closed path of length $k$, or a closed $k$-path, by $\bar{P}_{k}$.

Pictorially, we view a closed path of length $k$ as follows:

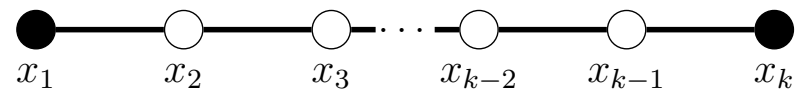

Proposition 15. Let $H=H^{1} \cup H^{2}$. If $H^{2}$ is bipartite and $H$ does not contain a copy of $K_{2}^{\{1,2\}}$ and $H$ contains a closed path of length $2 k$, then $\pi(H)=\frac{9}{8}$.

Proof. First, we will give a construction giving us the lower bound. For any $n \in \mathbb{N}$ let $G_{n}$ have vertex set $[n]$. Partition the vertices of $G_{n}$ into two sets $X$ and $\bar{X}$ where $|X|=\frac{3 n}{4}$ and $|\bar{X}|=\frac{n}{4}$. Let

$$
E(G)=\{\{x\}: x \in X\} \cup\{\{x, \bar{x}\}: x \in X \text { and } \bar{x} \in \bar{X}\} .
$$

It is clear that $G_{n}$ contains no closed paths of length $2 k$ when $k \geqslant 1$. Also,

$$
\begin{aligned}
\lim _{n \rightarrow \infty} h_{n}\left(G_{n}\right) & =\lim _{n \rightarrow \infty} \frac{|X|}{\left(\begin{array}{l}
n \\
1
\end{array}\right)}+\frac{|X| \cdot|\bar{X}|}{\left(\begin{array}{l}
n \\
2
\end{array}\right)} \\
& =\lim _{n \rightarrow \infty} \frac{3}{4}+\frac{\frac{3}{16} n^{2}}{\left(\begin{array}{c}
n \\
2
\end{array}\right)} \\
& =\frac{3}{4}+\frac{3}{8}=\frac{9}{8} .
\end{aligned}
$$

Thus $\pi(H) \geqslant \frac{9}{8}$ for any $H$ containing a closed path of length $2 k$ for any $k \geqslant 1$.

Since $H^{2}$ is bipartite, and $H^{2}$ does not contain a copy of $K_{2}^{\{1,2\}}$, then $H$ is contained in a blow-up of a closed 4-path. To see this, note that there is a bipartition of the vertices of $H, V(H)=A \cup B$, (with respect to the 2-edges in $H$ ). Furthermore, we can partition $A$ into $A_{1} \cup A_{2}$ where $v \in A_{1}$ if $\{v\} \in E(H)$ and $v \in A, v \in A_{2}$ if $v \in A \backslash A_{1}$. And similarly partition $B$ into $B_{1} \cup B_{2}$ with $v \in B_{1}$ if $\{v\} \in E(H)$ and $v \in B$. Then note that there are no edges from $A_{1}$ to $B_{1}$ since $H$ contains no copy of $K_{2}^{\{1,2\}}$. So $H \subset \bar{P}_{4}\left(\max \left\{\left|A_{1}\right|,\left|A_{2}\right|,\left|B_{1}\right|,\left|B_{2}\right|\right)-\right.$ a blow-up of $\bar{P}_{4}$. Below is a graphical representation of $H$, illustrating that $H$ is contained in a blow-up of $\bar{P}_{4}$.

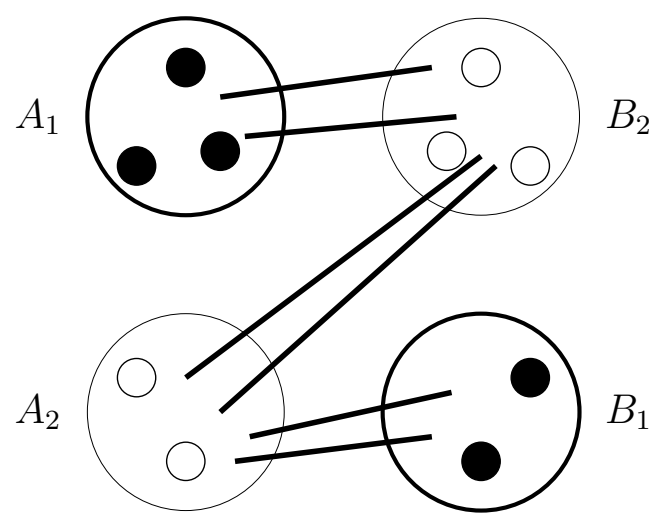


Since $\pi(H) \leqslant \pi\left(\bar{P}_{4}(s)\right)=\pi\left(\bar{P}_{4}\right)$ we need only show that $\pi\left(\bar{P}_{4}\right) \leqslant \frac{9}{8}$. Let $G_{n}$ be a family of $\bar{P}_{4}$-free graphs such that $h_{n}\left(G_{n}\right)=\pi_{n}\left(\bar{P}_{4}\right)$. Partition the vertices of $G_{n}$ as follows:

$$
\begin{aligned}
X_{n} & =\left\{v:\{v\} \in E\left(G_{n}\right)\right\}, \\
Y_{n} & =\left\{v:\{v\} \notin E\left(G_{n}\right) \text { and } \exists x_{1} \neq x_{2} \in X_{n} \text { with }\left\{x_{1}, v\right\},\left\{x_{2}, v\right\} \in E\left(G_{n}\right)\right\}, \\
Z_{n} & =V(G) \backslash\left(X_{n} \cup Y_{n}\right) .
\end{aligned}
$$

Let us say that $\left|X_{n}\right|=x n,\left|Y_{n}\right|=y n$ and hence $\left|Z_{n}\right|=(1-x-y) n$.

First, note that $E(G) \cap\left(\begin{array}{c}Y_{n} \\ 2\end{array}\right)=\emptyset$. Otherwise, since each vertex in $Y_{n}$ has at least 2 neighbors in $X_{n}, G_{n}$ would contain a closed path of length 4 . Also, each vertex in $Z_{n}$ has at most 1 neighbor in $X_{n}$. It follows that

$$
\begin{aligned}
\pi\left(\bar{P}_{4}\right) & =\lim _{n \rightarrow \infty} \pi_{n}\left(\bar{P}_{4}\right) \\
& =\lim _{n \rightarrow \infty} h_{n}\left(G_{n}\right) \\
& \leqslant \lim _{n \rightarrow \infty} \frac{\left|X_{n}\right|}{\left(\begin{array}{c}
n \\
1
\end{array}\right)}+\frac{\left|X_{n}\right| \cdot\left|Y_{n}\right|}{\left(\begin{array}{c}
n \\
2
\end{array}\right)}+\frac{\left|Y_{n}\right| \cdot\left|Z_{n}\right|}{\left(\begin{array}{c}
n \\
2
\end{array}\right)}+\frac{\left(\begin{array}{c}
\left|Z_{n}\right| \\
2
\end{array}\right)}{\left(\begin{array}{c}
n \\
2
\end{array}\right)}+\frac{\left|Z_{n}\right|}{\left(\begin{array}{c}
n \\
2
\end{array}\right)} \\
& \leqslant \lim _{n \rightarrow \infty} \frac{x n}{\left(\begin{array}{c}
n \\
1
\end{array}\right)}+\frac{x y n^{2}}{\left(\begin{array}{c}
n \\
2
\end{array}\right)}+\frac{y(1-x-y) n^{2}}{\left(\begin{array}{c}
n \\
2
\end{array}\right)}+\frac{\frac{(1-x-y)^{2} n^{2}}{2}}{\left(\begin{array}{c}
n \\
2
\end{array}\right)}+\frac{(1-x-y) n}{\left(\begin{array}{c}
n \\
2
\end{array}\right)} \\
& \leqslant \max _{\substack{0 \leqslant x \leqslant 1 \\
0 \leqslant y \leqslant 1-x}} x+2 x y+2 y(1-x-y)+(1-x-y)^{2} \\
& =\frac{9}{8} .
\end{aligned}
$$

The last inequality is an easy multivariate calculus exercise. It may be of interest to note that the maximum value of the function is obtained when $x=\frac{3}{4}$ and $y=\frac{1}{4}$. In this case $Z_{n}$ is empty. Since our upper bound matches the lower bound, we have the desired result.

Proposition 16. Let $H=H^{1} \cup H^{2}$. If $H^{2}$ is bipartite and $H^{2}$ does not contain a closed $2 k$-path for any $k \geqslant 1$, then $\pi(H)=1$.

Proof. First, since $|R(H)|=2$ we have, trivially, that $\pi(H) \geqslant 1$. Since $H$ contains no path of length $2 k$ for any $k \geqslant 1$ it must be the case that $H$ is contained in a blow-up of a chain $C^{\{1,2\}}=\{\{x\},\{x, y\}\}$. This is most clearly seen by again, considering the previous illustration. The difference is, in this case, $B_{1}$ (or $A_{1}$ ) is empty. 


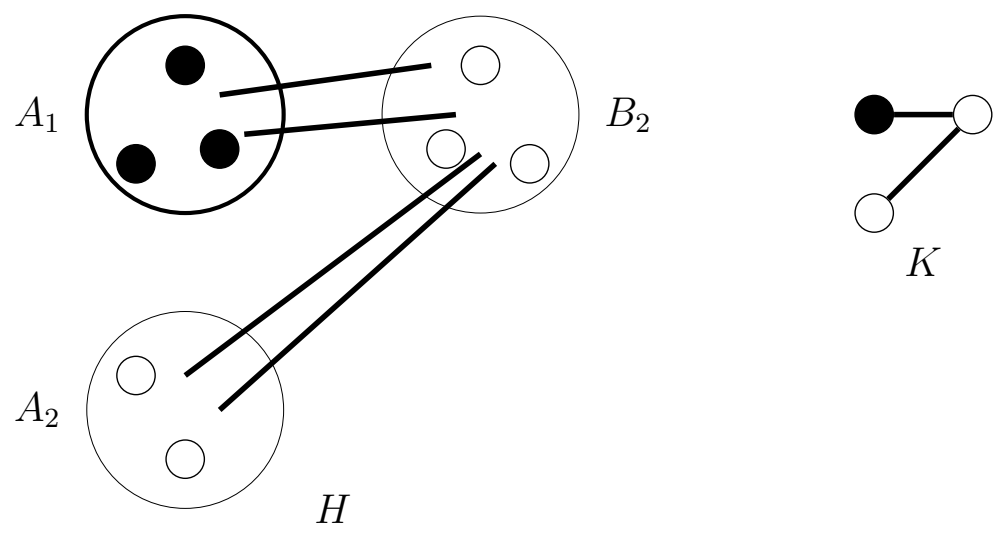

It is clear that $H$ is contained in a blow-up of $K$ where

$$
K=\{\{x\},\{x, y\},\{y, z\}\} \subseteq C^{\{1,2\}}(2,1)=\{\{x\},\{z\},\{x, y\},\{z, y\}\} .
$$

It follows that $\pi(H) \leqslant \pi\left(C^{\{1,2\}}\right)=1$.

The combination of these propositions completely determines $\pi(H)$ when $R(H)=$ $\{1,2\}$. The results are summarized by the following theorem.

Theorem 17. For any hypergraph $H$ with $R(H)=\{1,2\}$, we have

$$
\pi(H)= \begin{cases}2-\frac{1}{\chi\left(H^{2}\right)-1} & \text { if } H^{2} \text { is not bipartite; } \\ \frac{5}{4} & \text { if } H^{2} \text { is bipartite and } \min \left\{k: \bar{P}_{2 k} \subseteq H\right\}=1 \\ \frac{9}{8} & \text { if } H^{2} \text { is bipartite and } \min \left\{k: \bar{P}_{2 k} \subseteq H\right\} \geqslant 2 ; \\ 1 & \text { if } H^{2} \text { is bipartite and } \bar{P}_{2 k} \nsubseteq H \text { for any } k \geqslant 1 .\end{cases}
$$

\section{Degenerate hypergraphs}

Recall that a hypergraph $H$ is degenerate if $\pi(H)=|R(H)|-1$. For $k$-uniform hypergraph $H, H$ is degenerate if and only $H$ is $k$-partite. From Proposition 2 and Theorem 9, we have the following proposition.

Proposition 18. Suppose $H$ is a degenerate hypergraph. Then the following properties hold.

- Every subgraph of $H$ is degenerate.

- Every blow-up of $H$ is degenerate.

- Any subgraph of the blow-up of a flag is degenerate.

Note that every flag is a subgraph of some blow-up of a chain with the same edge type. Is every degenerate hypergraph a subgraph of some blow-up of a chain? The answer is yes for uniform hypergraphs and $\{1,2\}$-hypergraphs. This follows from Theorem 17, which 
completely determined $\pi(H)$ when $R(H)=\{1,2\}$, and from the fact that a $k$-uniform hypergraph is degenerate if and only if it is $k$-partite (a subgraph of a blow-up of a single edge). However, the answer in general is false. We will show that the following hypergraph $H_{1}$ with edge set $E\left(H_{1}\right)=\{\{1,2\},\{1,3\},\{2,3,4\}\}$ is degenerate.

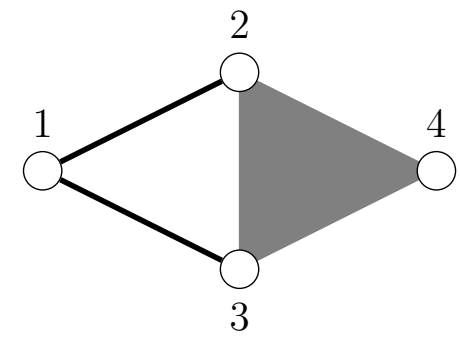

$H_{1}$ : A degenerate hypergraph not contained in the blow-up of a chain.

This result is a special case of the following theorem.

Definition 19. Let $H$ be a hypergraph containing some 2-edges. The 2-subdivision of $H$ is a new hypergraph $H^{\prime}$ obtained from $H$ by subdividing each 2-edge simultaneously. Namely, if $H$ contains $t$ 2-edges, add $t$ new vertices $x_{1}, x_{2}, \ldots, x_{t}$ to $H$ and for $i=1,2, \ldots, t$ replace the 2-edge $\left\{u_{i}, v_{i}\right\}$ with $\left\{u_{i}, x_{i}\right\}$ and $\left\{x_{i}, v_{i}\right\}$.

Theorem 20. Let $H^{\prime}$ be the 2-subdivision of $H$. If $H$ is degenerate, then so is $H^{\prime}$.

For example, $H_{1}$ can be viewed as the 2-division of the chain $C^{\{2,3\}}$. Since any chain is degenerate, so is $H_{1}$. To prove this theorem, we need a lemma on graphs, which has independent interest.

Definition 21. Let $G$ be any simple graph. Then $G^{(2)}$, a variation of the square of $G$, will be defined as the following simple graph:

- $V\left(G^{(2)}\right):=V(G)$,

- $E\left(G^{(2)}\right):=\{\{u, v\}: \exists w \in V(G)$ with $\{u, w\},\{v, w\} \in E(G)\}$.

Note that an edge of $G$ may or may not be an edge of $G^{(2)}$. For example, if $G$ is the complete graph, then $G^{(2)}$ is also the complete graph. However, if $G$ is a complete bipartite graph with partite set $V_{1} \cup V_{2}$, then $G^{(2)}$ is the disjoint union of two complete graphs on $V_{1}$ and $V_{2}$. In this case, $G^{(2)}$ is the complement graph of $G$. We also note that $G^{(2)}$ is the empty graph if and only if $G$ is a matching. Surprisingly, we have the following lemma on the difference of the number of edges in $G$ and $G^{(2)}$.

Lemma 22. For any simple graph $G$ on $n$ vertices,

$$
|E(G)|-\left|E\left(G^{(2)}\right)\right| \leqslant\left\lfloor\frac{n}{2}\right\rfloor .
$$


Furthermore, equality holds if and only if $G$ is the vertex-disjoint union of complete bipartite graphs of balanced part-size with at most one component having an odd number of vertices, i.e.

$$
G=K_{t_{1}, t_{1}} \cup K_{t_{2}, t_{2}} \cup \cdots \cup K_{t_{k}, t_{k}} \cup K_{\left\lfloor\frac{n}{2}\right\rfloor-\sum_{i=1}^{k} t_{i},\left\lceil\frac{n}{2}\right\rceil-\sum_{i=1}^{k} t_{i}},
$$

for some positive integers $t_{1}, t_{2}, \ldots, t_{k}$ satisfying $\sum_{i=1}^{k} t_{i} \leqslant\left\lfloor\frac{n}{2}\right\rfloor$.

Proof. First, we will show that inequality (7) holds for any forest. Let $G$ be a forest. Since $G$ is a forest, if $\{a, b\} \in E\left(G^{(2)}\right)$ then $a$ and $b$ have a unique common neighbor in $G$. Furthermore, given any vertex $c \in V(G)$, it follows that any pair of neighbors of $c$ is in $E\left(G^{(2)}\right)$. Thus we have

$$
\begin{aligned}
|E(G)|-\left|E\left(G^{(2)}\right)\right| & =\frac{1}{2} \sum_{v \in V(G)} \operatorname{deg}(v)-\sum_{v \in V(G)}\left(\begin{array}{c}
\operatorname{deg}(v) \\
2
\end{array}\right) \\
& =\sum_{v \in V(G)} \frac{1}{2} \operatorname{deg}(v)-\left(\begin{array}{c}
\operatorname{deg}(v) \\
2
\end{array}\right) \\
& =\sum_{v \in V(G)}-\frac{1}{2} \operatorname{deg}(v)^{2}+\operatorname{deg}(v) \\
& \leqslant \sum_{v \in V(G)} \frac{1}{2} \\
& =\frac{n}{2}
\end{aligned}
$$

The inequality above comes from the fact that $-\frac{1}{2} x^{2}+x \leqslant \frac{1}{2}$, attaining its maximum when $x=1$. Since $|E(G)|-\left|E\left(G^{(2)}\right)\right|$ is an integer, we have that

$$
|E(G)|-\left|E\left(G^{(2)}\right)\right| \leqslant\left\lfloor\frac{n}{2}\right\rfloor
$$

as claimed.

Now we will prove the statement $|E(G)|-\left|E\left(G^{(2)}\right)\right| \leqslant\left\lfloor\frac{|V(G)|}{2}\right\rfloor$ for general graphs using induction on the number of vertices. It holds trivially for $n=1,2$.

Assume that the statement holds for all graphs with fewer than $n$ vertices. Consider a graph $G$ with $n$ vertices. If $G$ is a forest, then the statement holds. Otherwise, $G$ contains a cycle. Choose $C_{g}$ to be a minimal cycle in $G$, i.e. one with no chords. If $G=C_{g}$, then $\mathrm{E}\left(C_{g}\right)-\mathrm{E}\left(C_{g}^{(2)}\right)=0$ if $g \neq 4$ or 2 if $g=4$. The statement holds.

Now assume $V(C) \subsetneq V(G)$. Let $V_{1}:=V(C)=\left\{x_{1}, x_{2}, \ldots, x_{g}\right\}$, where $x_{i}$ is adjacent to $x_{i+1}$, and let $V_{2}:=V(G) \backslash V_{1}=\left\{v_{1}, v_{2}, \ldots, v_{n-g}\right\}$.

The edges of $G$ can be partitioned into three parts: the induced graph $G\left[V_{1}\right]=C_{g}$, the induced graph $G\left[V_{2}\right]$, and the bipartite graph $G\left[V_{1}, V_{2}\right]$. Similarly, the edges of $G^{(2)}$ can be partitioned into three parts: $G^{(2)}\left[V_{1}\right]$, the induced graph $G^{(2)}\left[V_{2}\right]$, and the bipartite graph $G^{(2)}\left[V_{1}, V_{2}\right]$. Now we compare term by term. 
1. Note $\left|E\left(G^{(2)}\left[V_{1}\right]\right)\right| \geqslant\left|E\left(C_{g}^{(2)}\right)\right|$, and $\left|E\left(C_{g}^{(2)}\right)\right|=g$ if $g \neq 4$ or 2 . We have

$$
\left|E\left(G\left[V_{1}\right]\right)\right|-\left|E\left(G^{(2)}\left[V_{1}\right]\right)\right| \leqslant\left|E\left(C_{g}\right)\right|-\left|E\left(C_{g}^{(2)}\right)\right| \leqslant\left\lfloor\frac{g}{2}\right\rfloor .
$$

2. By inductive hypothesis, we have $\left|E\left(G\left[V_{2}\right]\right)\right|-\left|E\left(\left(G\left[V_{2}\right]\right)^{(2)}\right)\right| \leqslant\left\lfloor\frac{n-g}{2}\right\rfloor$. Combining with the fact $\left|E\left(G^{(2)}\left[V_{2}\right]\right) \geqslant\right| E\left(\left(G\left[V_{2}\right]\right)^{(2)}\right) \mid$, we have

$$
\left|E\left(G\left[V_{2}\right]\right)\right|-\left|E\left(G^{(2)}\left[V_{2}\right]\right)\right| \leqslant\left\lfloor\frac{n-g}{2}\right\rfloor .
$$

3. We claim $\left|E\left(G\left[V_{1}, V_{2}\right]\right)\right| \leqslant\left|E\left(G^{(2)}\left[V_{1}, V_{2}\right]\right)\right|$. We define a map

$$
f: E\left(G\left[V_{1}, V_{2}\right]\right) \rightarrow E\left(G^{(2)}\left[V_{1}, V_{2}\right]\right)
$$

as follows. For any edge $x_{i} v \in E(G)$ with $v \in V_{2}$ and $x_{i} \in V_{1}$, define $f\left(v x_{i}\right)=v x_{i+1}$ (with the convention $x_{g+1}=x_{1}$ ). Since $x_{i} v \in E(G)$ and $x_{i} x_{i+1} \in E(G)$, we have $v x_{i+1} \in E\left(G^{(2)}\right)$. The map $f$ is well-defined. We also observe that $f$ is an injective map. Thus

$$
\left|E\left(G\left[V_{1}, V_{2}\right]\right)\right| \leqslant\left|E\left(G^{(2)}\left[V_{1}, V_{2}\right]\right)\right| .
$$

Combining Equations (9), (10), and (11), we get

$$
|E(G)|-\left|E\left(G^{(2)}\right)\right| \leqslant\left\lfloor\frac{n-g}{2}\right\rfloor+\left\lfloor\frac{g}{2}\right\rfloor \leqslant\left\lfloor\frac{n}{2}\right\rfloor .
$$

The inductive step is finished.

Now we check when equality holds. It is straightforward to verify the sufficient condition; we omit the computation here.

Now we prove the necessary condition. Assume that $G$ has $k+1$ connected components $G_{1}, G_{2}, \ldots, G_{k+1}$. Then we have

$$
|E(G)|-\left|E\left(G^{(2)}\right)\right| \leqslant \sum_{i=1}^{k+1}\left(\left|E\left(G_{i}\right)\right|-\left|E\left(G_{i}^{(2)}\right)\right|\right) \leqslant \sum_{i=1}^{k+1}\left\lfloor\frac{\left|V\left(G_{i}\right)\right|}{2}\right\rfloor \leqslant\left\lfloor\frac{n}{2}\right\rfloor .
$$

If equality holds, then all but possibly one component has an even number of vertices. It remains to show each component is a balanced complete bipartite graph.

Without loss of generality, we assume $G$ is connected. If $G$ is a tree, then equality in Equation (8) either forces the degree of every vertex to be 1, or all the degrees are 1 with a single exceptional vertex of degree 2 . Since $G$ is assumed to be connected, $G$ is either $P_{2}=K_{1,1}$ or $P_{3}=K_{1,2}$.

Suppose that $G$ contains cycles, and the equalities hold in Equations (9), (10), and (11). First we show that $C_{4}$ is the only possible chordless cycle in $G$. Suppose not; let $C_{g}(g \neq 4)$ be a cordless cycle. We have $\left|E\left(C_{g}\right)\right|-\left|E\left(C_{g}^{(2)}\right)\right|=0$; which contradicts the 
assumption that equality holds in Equation (9). Thus $G$ is a bipartite graph. Furthermore,the equality in 10 forces each vertex $v$ to be connected to at least 2 vertices of $C_{4}$. Hence $G$ is 2-connected. Now $G$ must be a complete bipartite graph. Otherwise, say $u v$ is a nonedge crossing the partite sets. Since $G$ is 2-connected, there exists a cycle containing both $u$ and $v$. Let $C$ be such a cycle with minimum length; $C$ is cordless but not a $C_{4}$. Contradiction. Finally we show $G=K_{s t}$ is balanced. Note that

$$
|E(G)|-\left|E\left(G^{(2)}\right)\right|=s t-\left(\begin{array}{l}
s \\
2
\end{array}\right)-\left(\begin{array}{l}
t \\
2
\end{array}\right)=\frac{n}{2}-\frac{(s-t)^{2}}{2} \leqslant\left\lfloor\frac{n}{2}\right\rfloor .
$$

The equality holds if only if $s=t$ for even $n$ or $|s-t|=1$ for odd $n$. Because the equality holds at Equation (12), there is at most one odd component. So $G$ is balanced.

Proof of Theorem 20. We will prove by contradiction. Let $R:=R(H)=R\left(H^{\prime}\right)$ be the common set of edge types of $H$ and $H^{\prime}$. Suppose that $H^{\prime}$ is not degenerate, then $\pi\left(H^{\prime}\right)>$ $|R|-1+\epsilon$ for some $\epsilon>0$. Thus, there exists an $n_{0}$ satisfying $\pi_{n}\left(H^{\prime}\right)>|R|-1+\epsilon / 2$ for any $n \geqslant n_{0}$. Let $G_{n}^{R}$ be a $H^{\prime}$-free hypergraph with $\pi_{n}(G)>|R|-1+\epsilon / 2$. Define a new hypergraph $G_{n}^{\prime}$ over the same vertex set of $G$ with a new edge set $E\left(G_{n}^{\prime}\right)=$ $\left[E\left(G_{n}\right) \backslash E\left(G_{n}^{2}\right)\right] \cup E\left(\left(G_{n}^{2}\right)^{(2)}\right)$. The hypergraph $G_{n}^{\prime}$ is obtained from $G_{n}$ by replacing all 2-edges by the edges in its square graph while keeping all the edges of other types. By Lemma 22, we have

$$
h_{n}\left(G_{n}^{\prime}\right) \geqslant h_{n}(G)-\frac{\left\lfloor\frac{n}{2}\right\rfloor}{\left(\begin{array}{l}
n \\
2
\end{array}\right)} \geqslant|R|-1+\epsilon / 2-\frac{1}{n} .
$$

Suppose that $H$ has $t$ 2-edges. Since $H$ is degenerate, so is the blow-up hypergraph $H(t+1)$. For sufficiently large $n, G_{n}^{\prime}$ contains a subhypergraph $H(t+1)$. By the definition of $G^{\prime}$, for every copy of $H \subseteq H\left(t+1\right.$ ) and every 2-edge $u_{i} v_{i}$ (for $1 \leqslant i \leqslant t$ ) of $H$, there exists a vertex $x_{i}:=x_{i}\left(u_{i}, v_{i}\right)$ satisfying $u_{i} x_{i}$ and $v_{i} x_{i}$ are 2-edges of $G$. Our goal is to force $x_{1}, x_{2}, \ldots, x_{t}$ to be distinct from the vertices of $H$ and from each other. This can be done by a greedy algorithm. Suppose that the vertices of $H$ are listed by $y_{1}, y_{2}, y_{3}, \ldots$, and so on. Each vertex $y_{i}$ has $t+1$ copies in $H(t+1)$. For $i=1,2,3, \ldots$, select a vertex $y_{i}^{\prime}$ from the $t+1$ copies of $y_{i}$ so that $y_{i}^{\prime}$ is not the same vertex as $x_{j}\left(u_{j}, v_{j}\right)$ for some 2-edge $u_{j} v_{j}$ where $u_{j}, v_{j}$ have been selected. This is always possible since $H$ has only $t 2$-edges. Thus, we found a copy of $H^{\prime}$ as a subgraph of $G$. Contradiction.

It remains an open question to classify all non-degenerate hypergraphs.

In the remainder of this section, we generalize the following theorem due to Erdős [14] on the Turán density of complete $k$-partitite $k$-uniform hypergraphs.

Theorem (Erdös [14]): Let $K_{k}^{(k)}\left(s_{1}, \ldots, s_{k}\right)$ be the complete $k$-partite $k$-uniform hypergraph with partite sets of size $s_{1}, \ldots, s_{k}$. Then any $K_{k}^{(k)}\left(s_{1}, \ldots, s_{k}\right)$-free $r$-uniform hypergraph can have at most $O\left(n^{k-\delta}\right)$ edges, where $\delta=\left(\prod_{i=1}^{k-1} s_{i}\right)^{-1}$.

We have the following theorem. 
Theorem 23. Let $L\left(s_{1}, s_{2}, \ldots, s_{v(L)}\right)$ be a blow-up of a flag $L^{R}$, we have

$$
\pi_{n}\left(L\left(s_{1}, s_{2}, \ldots, s_{v(L)}\right)\right)=r-1+O\left(n^{-\delta}\right),
$$

where

$$
\delta=\frac{\max \left\{s_{i}: 1 \leqslant i \leqslant v(L)\right\}}{\prod_{i=1}^{v(L)} s_{i}} .
$$

Using the concept of $H$-density, we can say a lot more about avoiding a blow-up of any hypergraph $H$.

Given two hypergraphs $H$ and $G$ with the same edge-type $R(H)=R(G)$, the density of $H$ in $G$, denoted by $\mu_{H}(G)$, is defined as the probability that a random injective map $f: V(H) \rightarrow V(G)$ satisfies $H \stackrel{f}{\hookrightarrow} G$ (i.e. $f$ maps $H$ to a labeled copy of $H$ in $G$ ). We have the following theorem.

Theorem 24. For a fixed hypergraph $H$ on $m$ vertices and $m$ positive integers $s_{1}, s_{2}, \ldots, s_{m}$, let $H\left(s_{1}, s_{2}, \ldots, s_{m}\right)$ be the blow-up of $H$. For sufficiently large $n$ and any hypergraph $G$ on $n$ vertices with edge type $R(G)=R(H)$, if $G$ contains no subgraph $H\left(s_{1}, s_{2}, \ldots, s_{m}\right)$, then

$$
\mu_{H}(G)=O\left(n^{-\delta}\right)
$$

where

$$
\delta=\frac{\max \left\{s_{i}: 1 \leqslant i \leqslant m\right\}}{\prod_{i=1}^{m} s_{i}} .
$$

Proof. We will prove by contradiction. We assume $\mu_{H}(G) \geqslant C n^{-\delta}$ for some constant $C$ to be chosen later. By reordering the vertices of $H$, we can assume $s_{1} \leqslant s_{2} \leqslant \cdots \leqslant s_{m}$. We assume $n$ is divisible by $m$ by adding some isolated vertices. Consider a random $m$-partition of $V(G)=V_{1} \cup V_{2} \cup \cdots \cup V_{m}$ where each part has size $\frac{n}{m}$. For any $m$-set $S$ of $V(G)$, we say $S$ is a transversal (with respect to this partition of $V(G)$ ) if $S$ intersects each $V_{i}$ exactly once. The probability that $S$ is transversal is given by $\frac{\left(\frac{n}{m}\right)^{m}}{\left(\begin{array}{c}n \\ m\end{array}\right)}$.

We say a labeled copy of $H$ in $G, f(H)$, is transversally ordered if $f\left(v_{i}\right) \in V_{i}$ for each vertex $v_{i}$ of $H$. Clearly, the probability that $f(H)$ is transversally ordered is exactly $\frac{1}{m !}$ of the probability that $f(H)$ is a transversal. By the definition of $\mu_{H}(G), G$ contains $\mu_{H}(G)\left(\begin{array}{c}n \\ m\end{array}\right) m$ ! labeled copies of $H$. Thus, the expected number of transversally ordered copies of $H$ is

$$
\mu_{H}(G)\left(\frac{n}{m}\right)^{m} \geqslant \frac{C}{m^{m}} n^{m-\delta} .
$$

There exists a partition so that the number of transversally ordered copies of $H$ is at least $C m^{-m} n^{m-\delta}$. Now we fix this partition $[n]=V_{1} \cup \cdots \cup V_{m}$.

For $t_{i} \in\left\{1, s_{i}\right\}$ with $i=1,2 \ldots, m$, let $f\left(t_{1}, t_{2}, \ldots, t_{m}\right)$ count the number of labeled copies of $H^{\prime}=H\left(t_{1}, t_{2}, \ldots, t_{m}\right)$ such that the first $t_{1}$ vertices of $H^{\prime}$ are in $V_{1}$, the second $t_{2}$ vertices of $H^{\prime}$ are in $V_{2}$, and so on.

Claim (a): For $0 \leqslant l \leqslant m-1$, we have

$$
f\left(s_{1}, \ldots, s_{l}, 1, \ldots, 1\right) \geqslant(1-o(1))\left(\begin{array}{c}
n / m \\
s_{1}
\end{array}\right)\left(\begin{array}{c}
n / m \\
s_{2}
\end{array}\right) \ldots\left(\begin{array}{c}
n / m \\
s_{l}
\end{array}\right)\left(\frac{n}{m}\right)^{m-l}\left(C n^{-\delta}\right)^{s_{1} s_{2} \cdots s_{l}} .
$$


We prove claim (a) by induction on $l$. For the initial case $l=0$, the claim is trivial since $f(1,1, \ldots, 1)$ counts the number of transversally ordered copies of $H$. We have

$$
f(1,1, \ldots, 1) \geqslant C n^{-\delta}\left(\frac{n}{m}\right)^{m} .
$$

Thus the statement holds for $l=0$.

Now we assume claim (a) holds for $l>0$. Consider the case $l+1$, for some $l \geqslant 0$. For any $S \in\left(\begin{array}{c}V_{1} \\ s_{1}\end{array}\right) \times \cdots \times\left(\begin{array}{c}V_{l} \\ s_{l}\end{array}\right) \times\left(\begin{array}{c}V_{l+2} \\ 1\end{array}\right) \times \cdots \times\left(\begin{array}{c}V_{m} \\ 1\end{array}\right)$, let $d_{S}$ be the number of vertices $v$ in $V_{l+1}$ such that the induced subgraph of $H^{\prime}$ on $S \times\{v\}$ is $H\left(s_{1}, \ldots, s_{l}, 1, \ldots, 1\right)$. The number of the blow-up of $v$ into $s_{l+1}$ copies is simply $\left(\begin{array}{c}d_{S} \\ s_{l+1}\end{array}\right)$. Thus, we have

$$
\begin{gathered}
f\left(s_{1}, \ldots, s_{l}, 1,1, \ldots, 1\right)=\sum_{S} d_{S} \\
f\left(s_{1}, \ldots, s_{l}, s_{l+1}, 1, \ldots, 1\right)=\sum_{S}\left(\begin{array}{c}
d_{S} \\
s_{l+1}
\end{array}\right) .
\end{gathered}
$$

Let $\bar{d}_{l}$ be the average of $d_{S}$. By equation (14) and the inductive hypothesis, we have

$$
\bar{d}_{l}=\frac{\sum_{S} d_{S}}{\left(\begin{array}{c}
n / m \\
s_{1}
\end{array}\right) \ldots\left(\begin{array}{c}
n / m \\
s_{l}
\end{array}\right)\left(\frac{n}{m}\right)^{m-l-1}} \geqslant(1-o(1)) \frac{n}{m}\left(C n^{-\delta}\right)^{s_{1} s_{2} \cdots s_{l}}
$$

Applying the convex inequality, we have

$$
\begin{aligned}
& f\left(s_{1}, \ldots, s_{l}, s_{l+1}, 1, \ldots, 1\right) \\
= & \sum_{S}\left(\begin{array}{c}
d_{S} \\
s_{l+1}
\end{array}\right) \\
\geqslant & \left(\begin{array}{c}
n / m \\
s_{1}
\end{array}\right) \ldots\left(\begin{array}{c}
n / m \\
s_{l}
\end{array}\right)\left(\frac{n}{m}\right)^{m-l-1}\left(\begin{array}{c}
\bar{d}_{l} \\
s_{l+1}
\end{array}\right) \\
= & \left(1+O\left(\frac{1}{\bar{d}_{l}}\right)\right)\left(\begin{array}{c}
n / m \\
s_{1}
\end{array}\right) \ldots\left(\begin{array}{c}
n / m \\
s_{l+1}
\end{array}\right)\left(\frac{n}{m}\right)^{m-l-1}\left(C n^{-\delta}\right)^{s_{1} s_{2} \cdots s_{l+1}} .
\end{aligned}
$$

This concludes the proof of the claim.

Applying claim (a) with $l=m-1$, we get

$$
f\left(s_{1}, s_{2}, \ldots, s_{m-1}, 1\right) \geqslant(1+o(1))\left(\begin{array}{c}
n / m \\
s_{1}
\end{array}\right) \ldots\left(\begin{array}{c}
n / m \\
s_{m-1}
\end{array}\right) \frac{n}{m}\left(C n^{-\delta}\right)^{s_{1} s_{2} \cdots s_{m-1}} .
$$

For any $S \in\left(\begin{array}{c}V_{1} \\ s_{1}\end{array}\right) \times \cdots \times\left(\begin{array}{l}V_{m-1} \\ s_{m-1}\end{array}\right)$, let $d_{S}$ be the number of vertices $v$ in $V_{m}$ such that the induced subgraph $G[S \times\{v\}]$ contains a transversal ordered copy of $H\left(s_{1}, \ldots, s_{m-1}, 1\right)$. Since $G$ is $H\left(s_{1}, \ldots, s_{m}\right)$-free, we have $d_{S} \leqslant s_{m}$. It implies

$$
f\left(s_{1}, s_{2}, \ldots, s_{m-1}, 1\right)=\sum_{S} d_{S} \leqslant\left(\begin{array}{c}
n / m \\
s_{1}
\end{array}\right) \ldots\left(\begin{array}{c}
n / m \\
s_{m-1}
\end{array}\right) s_{m} .
$$

Choosing $C$ sufficiently large, say $C>\left(2 m s_{m}\right)^{1 /\left(s_{1} \cdots s_{m-1}\right)}$, Equations (17) and (18) contradict each other. 
Proof of Theorem 23. Let $r=|R|$ and consider a hypergraph $G:=G_{n}^{R}$ with

$$
h_{n}(G)=r-1+C n^{-\delta} .
$$

It suffices to show that $\mu_{H}(G) \geqslant C^{\prime} n^{-\delta}$.

Given a random permutation $\sigma$, let $X$ be the number of edges on a random full chain $\sigma(L)$. By the definition of the Lubell function, we have $h_{n}(G)=\mathrm{E}(X)$. Note $X$ only takes integer values $0,1, \ldots, r$. Since $\mathrm{E}(X)>r-1$, there is non-zero probability that $X=r$. In fact, we have

$$
\begin{aligned}
\mathrm{E}(X) & =\sum_{i=0}^{r} i \operatorname{Pr}(X=i) \\
& \leqslant r \operatorname{Pr}(X=r)+(r-1)(1-\operatorname{Pr}(X=r)) \\
& =r-1+\operatorname{Pr}(X=r) .
\end{aligned}
$$

Thus, we get

$$
\operatorname{Pr}(X=r) \geqslant \frac{C}{n^{\delta}}
$$

Every flag $\sigma(L)$ contributes an equal share of the probability of the event that $X=r$, namely,

$$
\frac{|A u t(L)|}{\left(\begin{array}{c}
n \\
v(L)
\end{array}\right) v(L) !} .
$$

Here $\operatorname{Aut}(L)$ is the automorphism of $L$. Thus, the number of such flags is at least

$$
\frac{C}{|A u t(L)| n^{\delta}}\left(\begin{array}{c}
n \\
v(L)
\end{array}\right) v(L) ! .
$$

It follows that $\mu_{H}(G) \geqslant C^{\prime} n^{-\delta}$, where $C^{\prime}:=C /|A u t(L)|$.

\section{Suspensions}

In this section we will investigate the relationship between $\pi(\mathcal{H})$ and $\pi(S(\mathcal{H}))$, where $S(\mathcal{H})$ is the suspension of hypergraphs in the family $\mathcal{H}$ (see Definition 4 ). We will further look at $\operatorname{limits}_{t \rightarrow \infty} \pi\left(\lim ^{t}(\mathcal{H})\right)$.

Definition 25. Given a graph $G$ with vertex set $v_{1}, \ldots, v_{n}$ the link hypergraph $G^{v_{i}}$ is the hypergraph with vertex set $V(G) \backslash\left\{v_{i}\right\}$ and edge set $E=\left\{F \backslash\left\{v_{i}\right\}: v_{i} \in F\right.$ and $F \in$ $E(G)\}$.

Proposition 26. For any family of hypergraphs $\mathcal{H}$ we have that $\pi(S(\mathcal{H})) \leqslant \pi(\mathcal{H})$. 
Proof. Let $G_{n}$ be a graph on $n$ vertices containing no copy of hypergraphs in $S(\mathcal{H})$ such that $h_{n}\left(G_{n}\right)=\pi_{n}(S(H))$. Say $V\left(G_{n}\right)=\left\{v_{1}, v_{2}, \ldots, v_{n}\right\}$. Note that for any $v_{i} \in V\left(G_{n}\right)$, we have that Lubell value of the corresponding link graph is

$$
h_{n-1}\left(G_{n}^{v_{i}}\right)=\sum_{F \in G_{n}, v_{i} \in F} \frac{1}{\left(\begin{array}{c}
n-1 \\
|F|-1
\end{array}\right)} .
$$

Also, note that $G_{n}^{v_{i}}$ contains no copy of $H \in \mathcal{H}$. If it did, then $S(H) \subset S\left(G_{n}^{v_{i}}\right) \subseteq G_{n}$; but $S(H)$ is not contained in $G_{n}$. Thus $h_{n-1}\left(G_{n}^{v_{i}}\right) \leqslant \pi_{n-1}(\mathcal{H})$. We then have the following:

$$
\begin{aligned}
\pi_{n}(S(H)) & =h_{n}\left(G_{n}\right) \\
& =\sum_{F \in E\left(G_{n}\right)} \frac{1}{\left(\begin{array}{c}
n \\
|F|
\end{array}\right)} \\
& =\sum_{F \in E\left(G_{n}\right)} \frac{1}{|F|} \sum_{v_{i} \in F} \frac{1}{\left(\begin{array}{c}
n \\
|F|
\end{array}\right)} \\
& =\sum_{i=1}^{n} \sum_{F \in E\left(G_{n}\right), v_{i} \in F} \frac{1}{|F|} \cdot \frac{1}{\left(\begin{array}{c}
n \\
|F|
\end{array}\right)} \\
& =\sum_{i=1}^{n} \sum_{F \in E\left(G_{n}\right), v_{i} \in F} \frac{1}{n} \cdot \frac{1}{\left(\begin{array}{c}
n-1 \\
|F|-1
\end{array}\right)} \\
& =\frac{1}{n} \sum_{i=1}^{n} h_{n-1}\left(G_{n}^{v_{i}}\right) \\
& \leqslant \frac{1}{n} \sum_{i=1}^{n} \pi_{n-1}(\mathcal{H}) \\
& =\pi_{n-1}(\mathcal{H}) .
\end{aligned}
$$

Thus, for any $n, \pi_{n}(S(\mathcal{H})) \leqslant \pi_{n-1}(\mathcal{H})$; taking the limit as $n \rightarrow \infty$ we get the result as claimed.

Corollary 27. If $H$ is degenerate, so is $S(H)$.

Conjecture 28. For all $H, \lim _{t \rightarrow \infty} \pi\left(S^{t}(H)\right)=|R(H)|-1$.

To conclude our paper, we prove a special case of this conjecture.

Theorem 29. Suppose that $H$ is a subgraph of the blow-up of a chain. Let $k_{1}$ be the minimum number in $R(H)$. Suppose $k_{1} \geqslant 2$, and $H^{\prime}$ is a new hypergraph obtained by adding finitely many edges of type $k_{1}-1$ arbitrarily to $H$. Then

$$
\lim _{t \rightarrow \infty} \pi\left(S^{t}\left(H^{\prime}\right)\right)=\left|R\left(H^{\prime}\right)\right|-1 .
$$


Proof. Without loss of generality, we can assume that $H$ is a blow-up of a chain and $V\left(H^{\prime}\right)=V(H)$. (This can be done by taking blow-up of $H$ and adding more edges.)

Suppose that $H$ has $v$ vertices and its edge type is $R(H):=\left\{k_{1}, k_{2}, \ldots, k_{r}\right\}$. Set $k_{0}:=k_{1}-1$ so that $R\left(H^{\prime}\right):=\left\{k_{0}, k_{1}, \ldots, k_{r}\right\}$. For convenience, we write $R$ for $R(H)$ and $R^{\prime}$ for $R\left(H^{\prime}\right)$, and

$$
\begin{aligned}
R+t & :=\left\{k_{1}+t, k_{2}+t, \ldots, k_{r}+t\right\}, \\
R^{\prime}+t & :=\left\{k_{0}+t, k_{1}+t, k_{2}+t, \ldots, k_{r}+t\right\} .
\end{aligned}
$$

For any small $\epsilon>0$, let $n_{0}=\left\lfloor\epsilon^{-t}\right\rfloor$. For any $n \geqslant n_{0}$ and any hypergraph $G_{n}^{R+t}$ with

$$
\pi_{n}(G)>|R(H)|-1+\epsilon=r+\epsilon,
$$

we will show $G$ contains a subhypergraph $S^{t}\left(H^{\prime}\right)$.

Take a random permutation $\sigma \in S_{n}$ and let $X$ be the number of edges in $G$ hit by the random full chain $C_{\sigma}$ :

$$
\emptyset \subset\{\sigma(1)\} \subset\{\sigma(1), \sigma(2)\} \cdots \subset\{\sigma(1), \sigma(2), \ldots, \sigma(i)\} \subset \cdots \subset[n] .
$$

We have

$$
\mathrm{E}(X)=\pi_{n}(G)>r+\epsilon .
$$

Since $X \leqslant r+1$, we have

$$
\begin{aligned}
\mathrm{E}(X) & =\sum_{i=0}^{r+1} i \operatorname{Pr}(X=i) \\
& \leqslant \sum_{i=0}^{r+1} r \operatorname{Pr}(X=i)+(r+1) \operatorname{Pr}(X=r+1) \\
& =r(1-\operatorname{Pr}(X=r+1))+(r+1) \operatorname{Pr}(X=r+1) \\
& \leqslant r+\operatorname{Pr}(X=r+1) .
\end{aligned}
$$

Thus, we get

$$
\operatorname{Pr}(X=r+1) \geqslant \mathrm{E}(X)-r>\epsilon .
$$

Recall that the density $\mu_{H}(G)$ is the probability that a random injective map $f: V(H) \rightarrow V(G)$ such that $H \stackrel{f}{\hookrightarrow} G$. We have

$$
\mu_{C^{R^{\prime}+t}}(G)=\operatorname{Pr}(X=r+1)>\epsilon .
$$

Every copy of the chain $C^{R^{\prime}+t}$ will pass through a set $A_{1} \in E^{k_{1}+t}(G)$. Let $\mu_{C^{R+t}, A_{1}}(G)$ be the conditional probability that a random injective map $f: V\left(C^{R+t}\right) \rightarrow V(G)$ satisfies $C^{R+t} \stackrel{f}{\hookrightarrow} G$ given that the chain $C^{R+t}$ passes through $A_{1}$. Let $d_{-}\left(A_{1}\right)$ be the number of sets $A_{0}$ satisfying $A_{0} \in E^{k_{0}+t}(G)$ and $A_{0} \subset A_{1}$. Then, we have

$$
\mu_{C^{R^{\prime}+t}}(G)=\frac{1}{\left(\begin{array}{c}
n \\
k_{1}+t
\end{array}\right)} \sum_{A_{1} \in E^{k_{1}+t(G)}} \mu_{C^{R+t}, A_{1}}(G) \cdot \frac{d_{-}\left(A_{1}\right)}{k_{1}+t} .
$$


Setting $\eta=\frac{\epsilon}{2}$, define a family

$$
\mathcal{A}=\left\{A_{1} \in E^{k_{1}+t}(G): \mu_{C^{R+t}, A_{1}}(G)>\eta \text { and } d_{-}\left(A_{1}\right)>\eta\left(k_{1}+t\right)\right\} .
$$

We claim $|\mathcal{A}|>\eta\left(\begin{array}{c}n \\ k_{1}+t\end{array}\right)$. Otherwise, we have

$$
\begin{aligned}
\mu_{C^{R^{\prime}+t}}(G) & =\frac{1}{\left(\begin{array}{c}
n \\
k_{1}+t
\end{array}\right)} \sum_{A_{1} \in E^{k_{1}+t(G)}} \mu_{C^{R+t}, A_{1}}(G) \cdot \frac{d_{-}\left(A_{1}\right)}{k_{1}+t} \\
& =\frac{1}{\left(\begin{array}{c}
n \\
k_{1}+t
\end{array}\right)} \sum_{A_{1} \in \mathcal{A}} \mu_{C^{R+t}, A_{1}}(G) \cdot \frac{d_{-}\left(A_{1}\right)}{k_{1}+t}+\frac{1}{\left(\begin{array}{c}
n \\
k_{1}+t
\end{array}\right)} \sum_{A_{1} \notin \mathcal{A}} \mu_{C^{R+t}, A_{1}}(G) \cdot \frac{d_{-}\left(A_{1}\right)}{k_{1}+t} \\
& \leqslant \eta+\eta<\epsilon .
\end{aligned}
$$

Contradiction.

A $k_{1}$-configuration is a pair $\left(S, A_{1}\right)$ satisfying $A_{1} \in \mathcal{A}, S=A_{1} \backslash\left\{i_{1}, i_{2}, \ldots, i_{k_{1}}\right\}$, and $A_{1} \backslash\left\{i_{j}\right\} \in E^{k_{0}+t}(G)$ for any $1 \leqslant j \leqslant k_{1}$.

For any $A_{1} \in \mathcal{A}$, the number of $S$ such that $\left(S, A_{1}\right)$ forms a $k_{1}$-configuration is at least

$$
\left(\begin{array}{c}
d_{-}\left(A_{1}\right) \\
k_{1}
\end{array}\right) \geqslant\left(\begin{array}{c}
\eta\left(k_{1}+t\right) \\
k_{1}
\end{array}\right)>\left(\frac{\eta}{2}\right)^{k_{1}}\left(\begin{array}{c}
k_{1}+t \\
k_{1}
\end{array}\right) .
$$

In the above inequality, we use the assumption $t>\frac{2}{\eta} k_{1}$.

By an averaging argument, there exists an $S$ so that the number of $k_{1}$-configurations $\left(S, A_{1}\right)$ is at least

$$
\frac{|\mathcal{A}|\left(\frac{\eta}{2}\right)^{k_{1}}\left(\begin{array}{c}
k_{1}+t \\
k_{1}
\end{array}\right)}{\left(\begin{array}{c}
n \\
t
\end{array}\right)} \geqslant \frac{\eta^{k_{1}+1}}{2^{k_{1}}}\left(\begin{array}{c}
n-t \\
k_{1}
\end{array}\right) .
$$

Now consider the link graph $G^{S}$. The inequality above implies

$$
\mu_{C^{R}}\left(G^{S}\right) \geqslant \frac{\eta^{k_{1}+2}}{2^{k_{1}}}
$$

This implies $G^{S}$ contains a blow-up of $C^{R}$. Thus $G^{S}$ has a subhypergraph $H$. By the definition of $k_{1}$-configuration, this $H$ can be extended to $H^{\prime}$ in $G^{S}$. In another words, $G$ contains $S^{t}\left(H^{\prime}\right)$.

\section{Connections to extremal poset problems}

As stated earlier, the Turán density of non-uniform hypergraphs is motivated by the extremal subset/poset problems.

Let $\mathcal{B}_{n}=\left(2^{[n]}, \subseteq\right)$ be the $n$-dimensional Boolean lattice. Under the partial relation $\subseteq$, any family $\mathcal{F} \subseteq 2^{[n]}$ can be viewed as a subposet of $\mathcal{B}_{n}$.

For posets $P=(P, \leqslant)$ and $P^{\prime}=\left(P^{\prime}, \leqslant^{\prime}\right)$, we say $P^{\prime}$ is a weak subposet of $P$ if there exists an injection $f: P^{\prime} \rightarrow P$ that preserves the partial ordering, meaning that whenever 
$u \leqslant \leqslant^{\prime} v$ in $P^{\prime}$, we have $f(u) \leqslant f(v)$ in $P$. If $P^{\prime}$ is not a weak poset of $P$, we say $P$ is $P^{\prime}$-free. The following problems originate from Sperner's theorem, which states that the largest antichain of $\mathcal{B}_{n}$ is $\left(\begin{array}{c}n \\ \left\lfloor\frac{n}{2}\right\rfloor\end{array}\right)$.

Extremal poset problems: Given a fixed poset $P$, what is the largest size of a $P$-free family $\mathcal{F} \subset \mathcal{B}_{n}$ ?

Let $\mathrm{La}(n, P)$ be the largest size of a $P$-free family $\mathcal{F} \subseteq \mathcal{B}_{n}$. The value of $\operatorname{La}(n, P)$ is known for only a few posets $P$. Let $\mathcal{P}_{k}$ be the (poset) chain of size $k$. Then $\operatorname{La}\left(n, \mathcal{P}_{2}\right)=$ $\left(\begin{array}{c}n \\ \left\lfloor\frac{n}{2}\right\rfloor\end{array}\right)$ by Sperner's theorem. Erdös [13] proved that $\mathrm{La}\left(n, \mathcal{P}_{k}\right)=\Sigma(n, k-1)$, where $\Sigma(n, k)$ is the sum of $k$ largest binomial coefficients. De Boinis-Katona-Swanepoel [9] proved $\mathrm{La}\left(n, \mathcal{O}_{4}\right)=\Sigma(n, 2)$. Here $\mathcal{O}_{4}$ is the butterfly poset $(A, B \subset C, D)$, or the crown poset of size 4.

The asymptotic value of $\mathrm{La}(n, P)$ has been discovered for various posets (see Table 1). Let $e(P)$ be the largest integer $k$ so that the family of $k$ middle layers of $\mathcal{B}_{n}$, denoted by $\mathcal{B}_{n}(k)$, is $P$-free. Griggs and Lu [27] originally conjecture that the limit

$$
\lim _{n \rightarrow \infty} \frac{\operatorname{La}(n, P)}{\left(\begin{array}{c}
n \\
\left\lfloor\frac{n}{2}\right\rfloor
\end{array}\right)}
$$

exists and is an integer. Mike Saks and Peter Winkler first observed this limit is $e(P)$ for all known values. Many authors believe that the following stronger version also holds.

Conjecture 30. For any fixed poset $P$,

$$
\lim _{n \rightarrow \infty} \frac{\operatorname{La}(n, P)}{\left(\begin{array}{c}
n \\
\left\lfloor\frac{n}{2}\right\rfloor
\end{array}\right)}=e(P) .
$$

We also use the notation $\pi(P)$ for the limit

$$
\lim _{n \rightarrow \infty} \frac{\operatorname{La}(n, P)}{\left(\begin{array}{c}
n \\
\left\lfloor\frac{n}{2}\right\rfloor
\end{array}\right)},
$$

where $P$ is a poset. The conjecture is based on the observation of several previous known results, which were obtained by Katona and others [5, 8, 9, 23, 28, 29, 49. We summarize some of the posets $P$, for which the conjecture has been verified in Table 1 .

The posets in Table 1 are far from complete. Let $\lambda_{n}(P)=\max \left\{h_{n}(\mathcal{F}): \mathcal{F} \subseteq\right.$ $2^{[n]}, P$-free $\}$. A poset $P$ is called uniform-L-bounded if $\lambda_{n}(P) \leqslant e(P)$ for all $n$. Griggs-Li [25] proved

$$
\mathrm{La}(n, P)=\sum_{i=\left\lfloor\frac{n-e(P)+1}{2}\right\rfloor}^{\left\lfloor\frac{n+e(P)-1}{2}\right\rfloor}\left(\begin{array}{l}
n \\
i
\end{array}\right)
$$

if $P$ is uniform-L-bounded. The uniform-L-bounded posets include $\mathcal{P}_{k}$ (for any $k \geqslant 1$ ), diamonds $\mathcal{D}_{k}$ (for $k \in\left[2^{m-1}-1,2^{m}-\left(\begin{array}{c}m \\ \left\lfloor\frac{m}{2}\right\rfloor\end{array}\right)-1\right]$ where $\left.m:=\left\lceil\log _{2}(k+2)\right\rceil\right)$, harps 


\begin{tabular}{|c|c|c|c|c|}
\hline$P$ & meaning & \begin{tabular}{|l|} 
Hasse \\
Diagram \\
\end{tabular} & $e(P)$ & References \\
\hline $\begin{array}{l}\text { fork } \\
\mathcal{V}_{r}\end{array}$ & $A<B_{1}, \ldots, B_{r}$ & & 1 & $\begin{array}{l}\text { [29 for } r=2 \\
\text { [8] for general } r\end{array}$ \\
\hline $\mathcal{N}$ & $A<B, B>C$, and $C<D$ & & 1 & 23 \\
\hline $\begin{array}{l}\text { butterfly } \\
\mathcal{O}_{4}\end{array}$ & $A<B, B>C, C<D$, and $A<D$ & & 2 & 9] \\
\hline $\begin{array}{l}\text { diamonds } \\
\mathcal{D}_{r}\end{array}$ & $\begin{array}{l}A<B_{1}, \ldots, B_{r}<C \\
\text { where } r=3,4,7,8,9,15,16, \ldots\end{array}$ & & varies & {$[26]$} \\
\hline $\begin{array}{l}\text { baton } \\
P_{k}(s, t)\end{array}$ & $\begin{array}{l}A_{1}, \ldots, A_{s}<B_{1}<B_{2}< \\
\cdots<B_{k-2}<C_{1}, \ldots, C_{t}\end{array}$ & $\stackrel{Y}{\Lambda}$ & $k-1$ & $\begin{array}{l}{[49 \text { for } s=1, k, t \geqslant 2} \\
{[27 \text { for } k, s, t \geqslant 2}\end{array}$ \\
\hline $\begin{array}{l}\text { tree } \\
T\end{array}$ & $\begin{array}{l}\text { A poset whose Hasse diagram is } \\
\text { a tree. Let } h(T) \text { be the height of } T \text {. }\end{array}$ & & $h(T)-1$ & $\begin{array}{l}\text { 27] for } h(T)=2 \\
\text { [4] for general cases }\end{array}$ \\
\hline $\begin{array}{l}\text { Crowns } \\
\mathcal{O}_{2 t}\end{array}$ & $\begin{array}{l}\text { A height- } 2 \text { poset, whose Hasse } \\
\text { diagram is a cycle } C_{2 t} \text {. }\end{array}$ & MI & 1 & $\begin{array}{l}{[27] \text { for even } t \geqslant 2} \\
\text { 36] for odd } t \geqslant 7\end{array}$ \\
\hline
\end{tabular}

Table 1: Conjecture 30 has been verified for various posets $P$.

$\mathcal{H}\left(l_{1}, l_{2}, \ldots, l_{k}\right)\left(\right.$ for $\left.l_{1}>l_{2}>\cdots>l_{k}\right)$, and other posets. Noteably, Griggs-Li [25] provides a method to construct large uniform-L-bounded posets from smaller uniform-L-bounded posets. There are infinitely many posets $P$ so that $\pi(P)=e(P)$ holds.

Although there is no counter example found yet for Conjecture 30 , some posets have resisted efforts to determine their $\pi$ value. The most studied, yet unsolved, poset is the diamond poset $\mathcal{D}_{2}$ (or $\mathcal{B}_{2}, Q_{2}$ in some papers) as shown in Figure 1. Griggs and Lu first observed $\pi\left(\mathcal{D}_{2}\right) \in[2,2.296]$. Axenovich, Manske, and Martin [1] came up with a new approach which improves the upper bound to 2.283. Griggs, Li, and Lu [26] further improved the upper bound to $2 \frac{3}{11} \approx 2.273$. Kramer-Martin-Young [34] recently proved $\pi\left(\mathcal{D}_{2}\right) \leqslant 2.25$. While it seems to be hard to prove the conjecture $\pi\left(\mathcal{D}_{2}\right)=2$, several groups of researchers have considered restricting the problem to three consecutive layers. Let $\operatorname{La}^{c}(n, P)$ be the largest size a $P$-free family $\mathcal{F} \subseteq \mathcal{B}_{n}$ such that $\mathcal{F}$ is in $e(P)+1$ consecutive layers. Let

$$
\pi^{c}(P)=\lim _{n \rightarrow \infty} \frac{\operatorname{La}^{c}(n, p)}{\left(\begin{array}{c}
n \\
\left\lfloor\frac{n}{2}\right\rfloor
\end{array}\right)},
$$

if the limit exists. Here is a weaker conjecture (of consecutive layers).

Conjecture 31. For any fixed poset $P, \pi^{c}(P)=e(P)$.

Axenovich-Manske-Martin [1] first proved $\pi^{c}\left(\mathcal{D}_{2}\right) \leqslant 2.207$; it was recently improved to 2.1547 (Manske-Shen [37]) and 2.15121 (Balogh-Hu-Lidický-Liu [3]).

We say a hypergraph $H$ represents a poset $P$ if the set of edges of $H$ (as a poset) extends $P$. For any fixed finite poset $P$, by the definition of $e(P)$, there exists an integer 

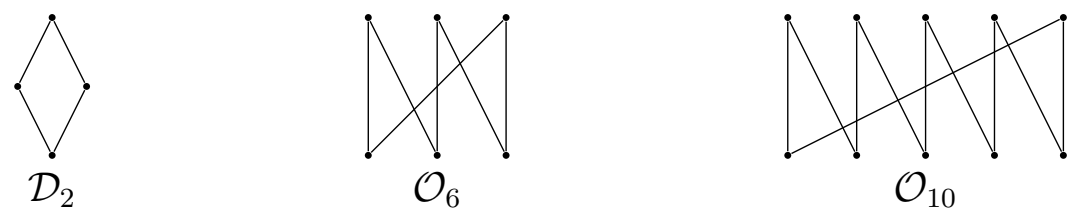

Figure 1: Three most wanted posets for Conjecture 30, $\mathcal{D}_{2}, \mathcal{O}_{6}$, and $\mathcal{O}_{10}$.

$n_{0}$ such that the middle $(e(p)+1)$-layers, denoted by $\mathcal{B}_{n_{0}}(e(P)+1)$, contains a hypergraph $H$ representing $P$.

Theorem 32. Let $n_{0}$ be any integer so that there is at least one hypergraph $H \subset$ $B_{n_{0}}(e(P)+1)$ representing $P$. Let $\mathcal{H}$ be a family of all hypergraphs in $\mathcal{B}_{n_{0}}(e(P)+1)$ representing $P$. Then

$$
\pi^{c}(P)=\lim _{t \rightarrow \infty} \pi\left(S^{t}(\mathcal{H})\right) .
$$

Proof. We first show that $\pi^{c}(P) \leqslant \pi\left(S^{t}(\mathcal{H})\right)$ for $t \geqslant 0$. Fix an integer $t$ and let $x:=$ $\pi\left(S^{t}(\mathcal{H})\right)$. For any $\epsilon>0$, there exists an $n_{1}$ so that $\pi_{n}\left(S^{t}(\mathcal{H})\right) \leqslant x+\epsilon$ for all $n \geqslant n_{1}$. We claim

$$
\pi^{c}(P) \leqslant x+3 \epsilon
$$

Otherwise, for any sufficiently large $n$, there exists a family $P$-free $\mathcal{F} \subset \mathcal{B}_{n}(e(P)+1)$ with $|\mathcal{F}|>(x+2 \epsilon)\left(\begin{array}{c}n \\ \left\lfloor\frac{n}{2}\right\rfloor\end{array}\right)$. Let $k_{0}$ be the smallest size of edges in $S^{t}(\mathcal{H})$. Let $k_{1}$ be the integer such that $\mathcal{F}$ is in the $k_{1}$-th to $\left(k_{1}+e(P)\right)$-th layers. Choose $n$ large enough so that $k_{1} \geqslant k_{0}$ and $n-k_{1}+k_{0} \geqslant n_{1}$. We observe that

$$
h_{n}(\mathcal{F}) \geqslant \frac{|F|}{\left(\begin{array}{c}
n \\
\left\lfloor\frac{n}{2}\right\rfloor
\end{array}\right)}>x+2 \epsilon .
$$

By the property of the Lubell function, $h_{n}(\mathcal{F})$ is the average of $h_{n+k_{0}-k_{1}}\left(\mathcal{F}_{S}\right)$ over all $S \in\left(\begin{array}{c}{[n]} \\ k_{1}-k_{0}\end{array}\right)$, where $\mathcal{F}_{S}$ is the link hypergraph over $S$. Therefore, there exists a set $S \in\left(\begin{array}{c}{[n]} \\ k_{1}-k_{0}\end{array}\right)$ so that $h_{n+k_{0}-k_{1}}\left(\mathcal{F}_{S}\right)>x+2 \epsilon$. Thus, $\mathcal{F}_{S}$ contains a subhypergraph in $S^{t_{0}}(\mathcal{H})$. In particular, $\mathcal{F}$ contains a subposet $P$.

Thus, we have

$$
\pi^{c}(P) \leqslant x+3 \epsilon
$$

Since this holds for any $\epsilon>0$, we have $\pi^{c}(P) \leqslant x$. Thus, we have

$$
\pi^{c}(P) \leqslant \pi\left(S^{t}(\mathcal{H})\right)
$$

Let $t$ approach the infinity. We have

$$
\pi^{c}(P) \leqslant \lim _{t \rightarrow \infty} \pi\left(S^{t}(\mathcal{H})\right) .
$$

Now we show that $\pi^{c}(P) \geqslant \lim _{t \rightarrow \infty} \pi\left(S^{t}(\mathcal{H})\right)$. Let $y=\lim _{t \rightarrow \infty} \pi\left(S^{t}(\mathcal{H})\right)$. For any $\epsilon>0$, we will construct a $P$-free family $\mathcal{F}_{k} \subset \mathcal{B}_{k}(e(P)+1)$ with $|\mathcal{F}|>y-\epsilon$ for any sufficiently large $k$. 
Choose $k\left(k \geqslant n_{0}\right)$ large enough so that

$$
\left(\begin{array}{l}
k \\
i
\end{array}\right) \geqslant\left(1-\frac{\epsilon}{2}\right)\left(\begin{array}{c}
k \\
\left\lfloor\frac{k}{2}\right\rfloor
\end{array}\right)
$$

for any $i \in\left[\left\lfloor\frac{k-e(P)}{2}\right\rfloor,\left\lfloor\frac{k+e(P)}{2}\right\rfloor\right]$. This is possible since $\lim _{k \rightarrow \infty} \frac{\left(\begin{array}{l}k \\ i\end{array}\right)}{\left(\begin{array}{l}k \\ \left\lfloor\frac{k}{2}\right\rfloor\end{array}\right)}=1$ for those $i$ 's.

Assume that $k$ and $n_{0}$ have the same parity. Set $t=\frac{k-n_{0}}{2}$ and $R$ be the set of consecutive $(e(P)+1)$ integers starting from $\left\lfloor\frac{k-e(P)}{2}\right\rfloor$. By the definition of $\pi\left(S^{t}(\mathcal{H})\right)$, for any sufficiently large $n$, there is a $S^{t}(\mathcal{H})$-free hypergraph $G_{n}^{R}$ with

$$
h_{n}(G) \geqslant \pi\left(S^{t}(\mathcal{H})\right)-\frac{\epsilon}{2} \geqslant y-\frac{\epsilon}{2} .
$$

For any $S \in\left(\begin{array}{c}{[n]} \\ k\end{array}\right)$, let $G[S]:=\{F: F \in E(G)$ and $F \subset S\}$. By the linearity of the Lubell value, $h_{n}(G)$ is the average of all $h_{k}(G[S])$ 's. Thus, there is a set $S \in\left(\begin{array}{c}{[n]} \\ k\end{array}\right)$ so that

$$
h_{k}(G[S]) \geqslant h_{n}(G) \geqslant y-\frac{\epsilon}{2} .
$$

View the edges of $G[S]$ as the family $\mathcal{F} \subset \mathcal{B}_{k}$. Then $\mathcal{F}$ is in the middle $(e(P)+1)$-levels of $\mathcal{B}_{k}$ by our choice of $t$. Moreover, $\mathcal{F}$ is $P$-free since all the representations in $\mathcal{H}$ are forbidden. Combining Equations (23) and (24), we have

$$
\begin{aligned}
|\mathcal{F}| & >\left(h_{k}(G[S])-\frac{\epsilon}{2}\right)\left(\begin{array}{c}
k \\
\left\lfloor\frac{k}{2}\right\rfloor
\end{array}\right) \\
& \geqslant(y-\epsilon)\left(\begin{array}{c}
k \\
\left\lfloor\frac{k}{2}\right\rfloor
\end{array}\right) .
\end{aligned}
$$

Thus we proved $\pi^{c}(P) \geqslant \lim _{t \rightarrow \infty} \pi\left(S^{t}(\mathcal{H})\right)$.

Corollary 33. For any poset $P$, suppose that $H$ is a hypergraph in the middle of $(e(P)+1)$ levels of some Boolean latttice representing $P$, then for any integer $t \geqslant 0$, we have

$$
\pi^{c}(P) \leqslant \pi\left(S^{t}(H)\right) .
$$

Corollary 34. Conjecture 28 implies Conjecture 31.

Note that the complete hypergraph $K_{2}^{\{0,1,2\}}$ has 4 edges $\emptyset,\{1\},\{2\},\{1,2\}$; which form the diamond poset $\mathcal{D}_{2}$. In particular, for any $t \geqslant 0$, we have

$$
\pi^{c}\left(\mathcal{D}_{2}\right) \leqslant \pi\left(S^{t}\left(K_{2}^{\{0,1,2\}}\right)\right) .
$$

This provides a possible way to improve the bounds of $\pi^{c}\left(\mathcal{D}_{2}\right)$. 


\section{Open questions}

For the convenience of the readers, we summarize all the conjectures raised in this paper. Conjecture 6 (See page 9 For any $k \geqslant 2, \pi\left(S^{k}\left(K_{2}^{\{1,2\}}\right)\right)=1+\max _{x \in(0,1)}\left\{x^{k+1}-x^{k+2}+\right.$ $\left.(1-x)^{k+1}-(1-x)^{k+2}\right\}$.

Let $f_{k}(x)=x^{k+1}-x^{k+2}+(1-x)^{k+1}-(1-x)^{k+2}$. Note that $f_{2}(x)$ reaches the maximum at $x=\frac{1}{2}$; the first case is that $\pi\left(S^{2}\left(K_{2}^{\{1,2\}}\right)\right)=1.125$. For $k \geqslant 3$, the maximum of $f_{k}(x)$ is roughly $\frac{1+o(1)}{e(k+1)}$. Here is a weaker version of Conjecture 6 .

Conjecture 6 For any $k \geqslant 3, \pi\left(S^{k}\left(K_{2}^{\{1,2\}}\right)\right)=1+O\left(\frac{1}{k}\right)$.

Conjecture 28 (See page 24) For all $R$-graph $H, \lim _{t \rightarrow \infty} \pi\left(S^{t}(H)\right)=|R(H)|-1$.

Conjecture 30 (See page 27) For any fixed poset $P, \lim _{n \rightarrow \infty} \frac{\operatorname{La}(n, P)}{\left(\begin{array}{l}n \\ \left\lfloor\frac{n}{2}\right\rfloor\end{array}\right)}=e(P)$.

Conjecture 31 (See page 28) For any fixed poset $P, \pi^{c}(P)=e(P)$.

\section{Acknowledgment}

We would like to thank the anonymous referee for the useful and detailed comments to prepare the final version.

\section{References}

[1] M. Axenovich, J. Manske, and R. Martin, $Q_{2}$-free families in the Boolean lattice, Order 30 (2013), 585-592.

[2] Rahil Baber and John Talbot, New Turán densities for 3-graphs, Elect. J. Combin., (2012), P22, 21p.

[3] J. Balogh, P. Hu, B. Lidický, and H. Liu, Upper bounds on the size of 4- and 6-cyclefree subgraphs of the hypercube, European J. Combin., 35 (2014), 75-85.

[4] B. Bukh, Set families with a forbidden poset, Elect. J. Combin. 16 (2009), R142, $11 \mathrm{p}$.

[5] T. Carroll and G. O. H. Katona, Bounds on maximal families of sets not containing three sets with $A \cup B \subset C, A \not \subset B$, Order 25 (2008), 229-236.

[6] F. Chung, L. Lu, An upper bound for the Turán number $t_{3}(n, 4)$. J. Combin. Theory Ser. A 87 (1999), no. 2, 381-389.

[7] D. Conlon, An extremal theorem in the hypercube. Electron. J. Combin. 17 (2010), \#R111.

[8] A. De Bonis and G. O. H. Katona, Largest families without an $r$-fork, Order 24 (2007), 181-191.

[9] A. De Bonis, G. O. H. Katona and K. J. Swanepoel, Largest family without $A \cup B \subseteq$ $C \cap D$, J. Combin. Theory (Ser. A) 111 (2005), 331-336. 
[10] D. de Caen, Extension of a theorem of Moon and Moser on complete subgraphs. Ars Combin. 16 (1983), 5-10.

[11] D. de Caen, The current status of Turán's problem on hypergraphs. Extremal problems for finite sets (Visegrád, 1991), 187-197, Bolyai Soc. Math. Stud., 3, János Bolyai Math. Soc., Budapest, 1994.

[12] D. de Caen, D. L. Kreher, S.P. Radziszowski, and W.H. Mills, On the coverings $t$-sets with $t+1$-sets: $C(9,5,4)$ and $C(10,6,5)$. Discrete Math. 92 (1991), 65-77.

[13] P. Erdős, On a lemma of Littlewood and Offord, Bull. Amer. Math. Soc. 51 (1945), 898-902.

[14] P. Erdős, On extremal problems of graphs and generalized graphs, Israel J. Math. 2 (1964), 183-190.

[15] P. Erdős, On the combinatorial problems which I would like to see solved, Combinatorica 1 (1981), 25-42.

[16] P. Erdős, M. Simonovits, Supersaturated graphs and hypergraphs. Combinatorica 3 (1983), 181-192.

[17] P. Frankl, Z. Füredi, A new generalization of the Erdős-Ko-Rado theorem, Combinatorica 3 (1983), 341-349.

[18] P. Frankl, Z. Füredi, An exact result for 3-graphs, Discrete Math. 50 (1984), 323-328.

[19] Z. Füredi, D. Mubayi and O. Pikhurko, Quadruple systems with independent neighborhoods, J. Comb. Theory Ser. A 115 (2008), 1552-1560.

[20] Z. Füredi and M. Simonovits, Triple systems not containing a Fano configuration, Combin. Probab. Comput. 14 (2005), 467-484.

[21] G. Giraud, Majoration du nombre de Ramsey ternaire-bicolore en $(4,4)$. C. R. Acad. Sci. Paris Sér. A-B 269 (1969), A620-A622.

[22] G. Giraud, Remarques sur deux problèmes extrémaux. (French) [Remarks on two extremal problems] Discrete Math. 84 (1990), no. 3, 319-321.

[23] J. R. Griggs and G. O. H. Katona, No four subsets forming an N, J. Combinatorial Theory (Ser. A) 115 (2008), 677-685.

[24] J. R. Griggs and W.-T. Li, The partition method for poset-free families, Journal of Combinatorial Optimization, 25, Issue 4, (2013), 587-596.

[25] J. R. Griggs and W.-T. Li, Poset-free families and Lubell-boundedness, arXiv:1208.4241.

[26] J. R. Griggs, W.-T. Li, and L. Lu, Diamond-free Families, Journal of Combinatorial Theory Ser. A, 119 (2012), 310-322.

[27] J. R. Griggs and L. Lu, On families of subsets with a forbidden subposet, Combinatorics, Probability, and Computing 18 (2009), 731-748.

[28] G. Katona, T. Nemetz, M. Simonovits, On a problem of Turán in the theory of graphs. Mat. Lapok 15 (1964), 228-238. 
[29] G. O. H. Katona and T. G. Tarján, Extremal problems with excluded subgraphs in the $n$-cube, in: M. Borowiecki, J. W. Kennedy, and M. M. Sysło (eds.) Graph Theory, Łagów, 1981, Lecture Notes in Math., 1018 84-93, Springer, Berlin Heidelberg New York Tokyo, 1983.

[30] P. Keevash, Hypergraph Turán Problems, Surveys in Combinatorics, Cambridge University Press, 2011, 80-140.

[31] P. Keevash, The Turán problem for hypergraphs on fixed size, Electron. J. Combin. 12 (2005), Note 11, 6 pp. (electronic).

[32] P. Keevash and B. Sudakov, The Turán number of the Fano plane, Combinatorica 25 (2005), 561-574.

[33] P. Keevash and B. Sudakov, On a hypergraph Turán problem of Frankl, Combinatorica 25 (2005), 673-706.

[34] L. Kramer, R. Martin, M. Young, On diamond-free subposets of the Boolean lattice, J. Combin. Theory Ser. A, 120, Issue 3, (2013), 545-560.

[35] N. W. Lemons, Turán Problems for Hypergraphs, dissertation, Central European University, Budapest, Hungary, (2008).

[36] Linyuan Lu, On crown-free families of subsets, J. Combin. Theory Ser. A., 126, (2014) 216-231.

[37] J. Manske and J. Shen, Three layer $Q_{2}$-free families in the Boolean lattice, Order $\mathbf{3 0}$, Issue 2 (2013), 585-592.

[38] K. Markström, Extremal graphs and bounds for the Turán density of the 4-uniform $\mathbf{K}_{5}$, Discrete Math. 309 no. 16, (2009), 5231-5234.

[39] D. Mubayi, On hypergraphs with every four points spanning at most two triples, Electron. J. Combin. 10 (2003), no. 1, Note 10, 4 pp. (electronic).

[40] D. Mubayi and Y. Zhao, Non-uniform Turán-type problems, J. Comb. Th. A, 111 (2004), 106-110.

[41] O. Pikhurko, Exact computation of the hypergraph Turán function for expanded complete 2-graphs, Accepted by J. Combin. Theory Ser. B publication suspended for an indefinite time, see http://www . math. cmu. edu/ pikhurko/Copyright.html.

[42] A. Razborov, On 3-hypergraphs with forbidden 4-vertex configurations, SIAM J. Disc. Math. 24 (2010), 946-963.

[43] A. Sidorenko, An analytic approach to extremal problems for graphs and hypergraphs. Extremal problems for finite sets (Visegrád, 1991), 423-455, Bolyai Soc. Math. Stud., 3, János Bolyai Math. Soc., Budapest, 1994.

[44] A. Sidorenko, On Turán numbers $T(n, 5,4)$ and numbers of monochromatic 4-cliques in 2-colored 3-graphs (in Russian). Voprosi Kibernetiki 64 (1980), 117-124.

[45] A. Sidorenko, The method of quadratic forms in a combinatorial problem of Turán. (in Russian) Vestnik Moskov. Univ. Ser. I Mat. Mekh. 76 (1982), no. 1, 3-6. 
[46] A. Sidorenko, Upper bounds for Turán numbers, J. Combin. Theory Ser. A 77 (1997), no. $1,134-147$.

[47] A. Sidorenko, What we know and what we do not know about Turán numbers. Graphs Combin. 11 (1995), no. 2, 179-199.

[48] J. Talbot, Chromatic Turán problems and a new upper bound for the Turán density of $\mathcal{K}_{4}^{-}$, Europ. J. Combin. 28 (2007), no. 8, 2125-2142.

[49] H. T. Thanh, An extremal problem with excluded subposets in the Boolean lattice, Order 15 (1998), 51-57.

[50] P. Turán, On an extremal problem in graph theory. Mat. Fiz. Lapok 48 (1941), 436-452. 